\title{
Pulsation and rotation in NGC 6811: the Kepler short-cadence stars
}

\author{
E. Rodríguez ${ }^{\oplus},{ }^{1 \star}$ L. A. Balona ${ }^{\oplus},{ }^{2 \star}$ M. J. López-González, ${ }^{1 \star}$ S. Ocando, ${ }^{1}$ \\ S. Martín-Ruiz ${ }^{1}$ and C. Rodríguez-López ${ }^{1}$ \\ ${ }^{1}$ Instituto de Astrofísica de Andalucía, CSIC, PO Box 3004, E-18080 Granada, Spain \\ ${ }^{2}$ South African Astronomical Observatory, PO Box 9, Observatory, Cape 2735, South Africa
}

Accepted 2019 November 6. Received 2019 November 6; in original form 2019 September 11

\begin{abstract}
We have analysed a selected sample of 36 Kepler short-cadence stars in the field of NGC 6811. The results reveal that all the targets are variable: two red giant stars with solar-like oscillations, 21 main-sequence pulsators (16 $\delta$ Scuti and five $\gamma$ Doradus stars), and 13 rotating variables. Three new $\gamma$ Doradus ( $\gamma$ Dor) variables (one is a hot $\gamma$ Dor star) are detected in this work together with five new rotating variables. An in-depth frequency analysis of the $\delta$ Scuti $(\delta$ Sct) and $\gamma$ Dor stars in the sample shows that the frequency spectra are very rich, in particular for the $\delta$ Sct-type variables. They present very dense frequency distributions and wide diversity in frequency patterns, even for stars being members of the cluster and with very similar location in the Hertzsprung-Russell (H-R) diagram. Rotational modulation is found for a high percentage of these main-sequence pulsating stars, which is an indication of stellar activity being common on the surfaces of these hot stars. In some cases, activity dominates the luminosity variations. Significant amplitude variability is also detected in at least some of the pulsation modes of highest amplitude in almost all the $\delta$ Sct stars. One of the $\delta$ Sct pulsators is a member of a binary system with the pulsating component tidally distorted. This system also shows strong stellar activity, including several flares that probably originate in the $\delta$ Sct component.
\end{abstract}

Key words: binaries: general-stars: oscillations -stars: rotation - stars: variables: $\delta$ Scutistars: variables: general.

\section{INTRODUCTION}

Our knowledge of stellar pulsation has rapidly advanced with the advent of space missions, particularly Kepler (Borucki et al. 2010; Koch et al. 2010), owing to the exceptional quality of the photometric time series. Kepler obtained almost uninterrupted photometry for over 150000 stars brighter than 16 mag, with a 29.4 min cadence over a 4 -yr time span. These are long-cadence (LC) observations. In addition, light curves with a cadence of about 1 min (short-cadence, SC, observations) are available for a few thousand stars, but these typically cover a time span of only a few months.

The $\gamma$ Doradus ( $\gamma$ Dor) and $\delta$ Scuti ( $\delta$ Sct) stars are A2-F5 dwarf and giants that pulsate with multiple frequencies. The $\delta$ Sct stars pulsate with typical frequencies in the range $5-50 \mathrm{~d}^{-1}$, while the $\gamma$ Dor stars pulsate in the $0-5 \mathrm{~d}^{-1}$ range. Both types of pulsating stars occupy a region of the instability strip that is an extension of the Cepheid instability strip towards lower luminosities. The $\gamma$ Dor stars populate a much smaller region near the cool end of the $\delta$ Sct instability strip.
Before the advent of high-precision photometry from space, it was thought that the high frequencies in $\delta$ Sct were driven by the $\kappa$ mechanism operating in the He II ionization zone (Pamyatnykh 2000). For $\gamma$ Dor stars, the driving mechanism was thought to be convective flux blocking near the base of the convective envelope (Guzik et al. 2000). Stars showing both low and high frequencies (the $\gamma$ Dor $/ \delta$ Sct hybrids) could be explained using time-dependent perturbation models (Dupret et al. 2004; Grigahcène et al. 2005). Besides, only a few hybrids were known, which appeared to be located between the $\delta$ Sct and $\gamma$ Dor instability regions.

Nowadays, it is known that at least 98 per cent of $\delta$ Sct stars are hybrids (Balona 2014a, 2018c). Therefore, it no longer makes sense to use the term 'hybrid'. Furthermore, less than half of the stars in the $\delta$ Sct instability strip seem to pulsate. Besides, the region where $\gamma$ Dor stars are to be found contains a mixture of $\gamma$ Dor, $\delta$ Sct, and constant stars (Balona 2018c). It seems that $\gamma$ Dor stars are just $\delta$ Sct stars in which the high frequencies are suppressed for a reason that is still unknown. The frequency content and distribution in $\delta$ Sct and $\gamma$ Dor stars varies widely even for stars with the same effective temperature and luminosity (Balona, Daszyńska-Daszkiewicz \& Pamyatnykh 2015; Balona 2018c).

Taking into account all the accumulated observational evidence, it is clear that we need a new description of the pulsation mechanisms. 
The $\kappa$ mechanism cannot account for low frequencies in $\delta$ Sct stars, and the convective flux blocking mechanism does not explain why $\delta$ Sct and $\gamma$ Dor stars occupy the same region of the instability strip. Neither mechanism can explain why there are so many constant stars in the instability region. Clearly, the frequencies are very sensitive to conditions in the outer atmosphere, which vary from star to star. The most sophisticated currently available models are those of Xiong et al. (2016). They suggest that convection plays an important role, even in the hottest stars, but the models are still unable to match the observations.

Another result derived from Kepler observations is that a large fraction of A and B stars show frequencies consistent with rotational modulation. Indeed, this is the most frequent variability encountered among these stars (Balona 2013, 2016). Such variability implies the existence of star-spots or other obscuration corotating with the star. This suggests the possible presence of surface magnetic fields, although this contradicts our current understanding of stars with radiative envelopes. The magnetic fields required for the production of spots on the Sun and cool stars are thought to be generated by the dynamo mechanism. Since this requires convection in the outer layers of a star, current models cannot account for the presence of magnetic fields in the radiative envelopes of $\mathrm{A}$ and $\mathrm{B}$ stars. The failure of current models to explain pulsations in $\delta$ Sct and $\gamma$ Dor stars, the presence of rotational modulation in A and B stars, the large fraction of constant stars in the $\delta$ Sct instability strip, etc. all suggest that a major revision of our current understanding of early-type stars is required.

For this reason, it is important to study $\delta$ Sct and $\gamma$ Dor stars in open clusters. One may presume that the ages and chemical composition of these stars are the same, and in this way we eliminate the effect of these variables. NGC 6811, with an age of about $1 \mathrm{Gyr}$ (Meibom et al. 2011), is one of four open clusters in the field of view of the original Kepler space mission, together with NGC 6866 (0.5 Gyr), NGC 6819 (2.5 Gyr), and NGC 6791 (9 Gyr). NGC 6811 is known to host several $\delta$ Sct and $\gamma$ Dor stars (van Cauteren et al. 2005; Luo et al. 2009; Debosscher et al. 2011; Uytterhoeven et al. 2011; Sandquist et al. 2016). There are also some red giants showing solar-like oscillations (Stello et al. 2011a,b; Corsaro et al. 2012), as well as rotational variables (Meibom et al. 2011; Nielsen et al. 2013; Reinhold, Reiners \& Basri 2013; McQuillan, Mazeh \& Aigrain 2014; Reinhold \& Gizon 2015). Photometric and spectroscopic studies to determine cluster membership are also available (Sanders 1971; Peña et al. 2011; Kharchenko et al. 2013; Zacharias et al. 2013; Dias et al. 2014; Molenda-Żakowicz et al. 2014).

The Kepler data base offers a unique opportunity to study, in depth, the frequency content for a sample of $\delta$ Sct and $\gamma$ Dor stars in NGC 6811. In order to extract the frequencies within the entire frequency domain of the $\delta$ Sct-type stars, it is necessary to use SC observations. Particular attention is devoted to peaks in the lowfrequency region, which may be explained by mechanisms other than pulsation. Additionally, the stability of the highest amplitude peaks will be examined for stars with sufficient observations.

\section{THE SAMPLE}

There are 32 stars observed in Kepler SC mode in the field of NGC 6811 within a radius $d<7.0$ arcmin from the cluster centre. This corresponds to primary field A of ground-based observations in Ocando et al. (in preparation). Four additional objects with available SC data located in the secondary adjacent field B were also included, bringing the total number of stars studied to 36 . The 36 selected stars are plotted in Fig. 1 in which the Padova stellar isochrone (Marigo et al. 2017) for $1 \mathrm{Gyr}, Z=0.012$, is shown. This age is in very good agreement with earlier estimates (e.g. Janes et al. 2013; Yontan et al. 2015).

Table 1 lists information for these stars including parameters derived from the literature. Effective temperatures are from Mathur et al. (2017), whereas the luminosities are determined either from Gaia Data Release 2 (DR2) parallaxes (Gaia Collaboration et al. 2016, 2018) (see Appendix A) or else from the Kepler Input Catalogue (KIC) radius (Mathur et al. 2017). Regarding membership, we follow the method of Curtis et al. (2019) in using the Gaia DR2 proper motions and parallaxes to identify cluster members. In general, there is good agreement with previous identifications from other authors. The majority of the targets are confirmed members of NGC 6811. Four stars in our sample do not have Gaia measurements: ID15, ID16, ID23, and ID35. ID23 is a probably member of the cluster (Sanders 1971; Peña et al. 2011), but there is no reliable information in the literature for the others.

Most of the sample stars are already known as main-sequence pulsators or rotational variables. Two stars, ID04 and ID10, are known to be red giants showing solar-like oscillations (Stello et al. 2011b; Molenda-Żakowicz et al. 2014). For a few stars no variability information exists in the literature.

Fig. 1 shows that all the $\delta$ Sct and $\gamma$ Dor stars in the sample lie well inside the observational region expected for both types of pulsators, except for ID34. This star is revealed as a likely new pulsator in the low-frequency domain, typical of the g-mode regime, but it is located in the hotter part of the Hertzsprung-Russell (H-R) diagram, outside the typical $\gamma$ Dor region. In fact, ID34 appears as the hottest star in our sample, probably belonging to the group of the rather rare hot $\gamma$ Dor stars discussed in Balona et al. (2016).

The location of ID36 is also interesting, with $\left(T_{\text {eff }}=7450 \mathrm{~K}\right.$, $\log L / \mathrm{L}_{\odot}=1.76$ ), which is consistent with the parameters derived from $u v b y \beta$ photometry in Peña et al. (2011) $\left(T_{\text {eff }}=7310 \mathrm{~K}\right.$, $\left.\log L / \mathrm{L}_{\odot}=1.53\right)$. It will be shown that ID36 is a $\delta$ Sct star in a binary system. The $\delta$ Sct star is tidally distorted and shows strong stellar activity, including flares.

The Kepler light curves are available in the Mikulski Archive for Space Telescope (MAST). ${ }^{1}$ All SC mode light curves for Q1Q17 were analysed. Column 6 of Table 1 lists the number of months available for each star in our sample. Kepler light curves are available in two forms: the Simple Aperture Photometry (SAP) or 'raw' light curves, where only basic corrections have been applied to the original fluxes, and the Pre-Search Data Conditioned (PDC) or corrected light curves, where instrumental effects have been removed. The PDC time series have been in constant evolution with new and more refined procedures being regularly applied. Hence, we preferred to use the PDC light curves available at the time of this analysis, although the SAP curves were also used for checking purposes. We additionally used our own procedure to correct for remaining jumps and outliers following the method described in Rodríguez et al. (2016). The LC data files were also used for checking purposes.

A preliminary investigation was performed for each star using the program package PERIOD04(Lenz \& Breger 2005) for visual inspection of the light curves and periodograms. All stars in the sample were found to be variable. The main variability types of each star are in very good agreement with those derived from earlier authors, as shown in Table 1. Three new $\gamma$ Dor pulsators (ID26, ID34, and ID35) are found together with five new rotating variables

\footnotetext{
${ }^{1}$ http://archive.stsci.edu/kepler
} 


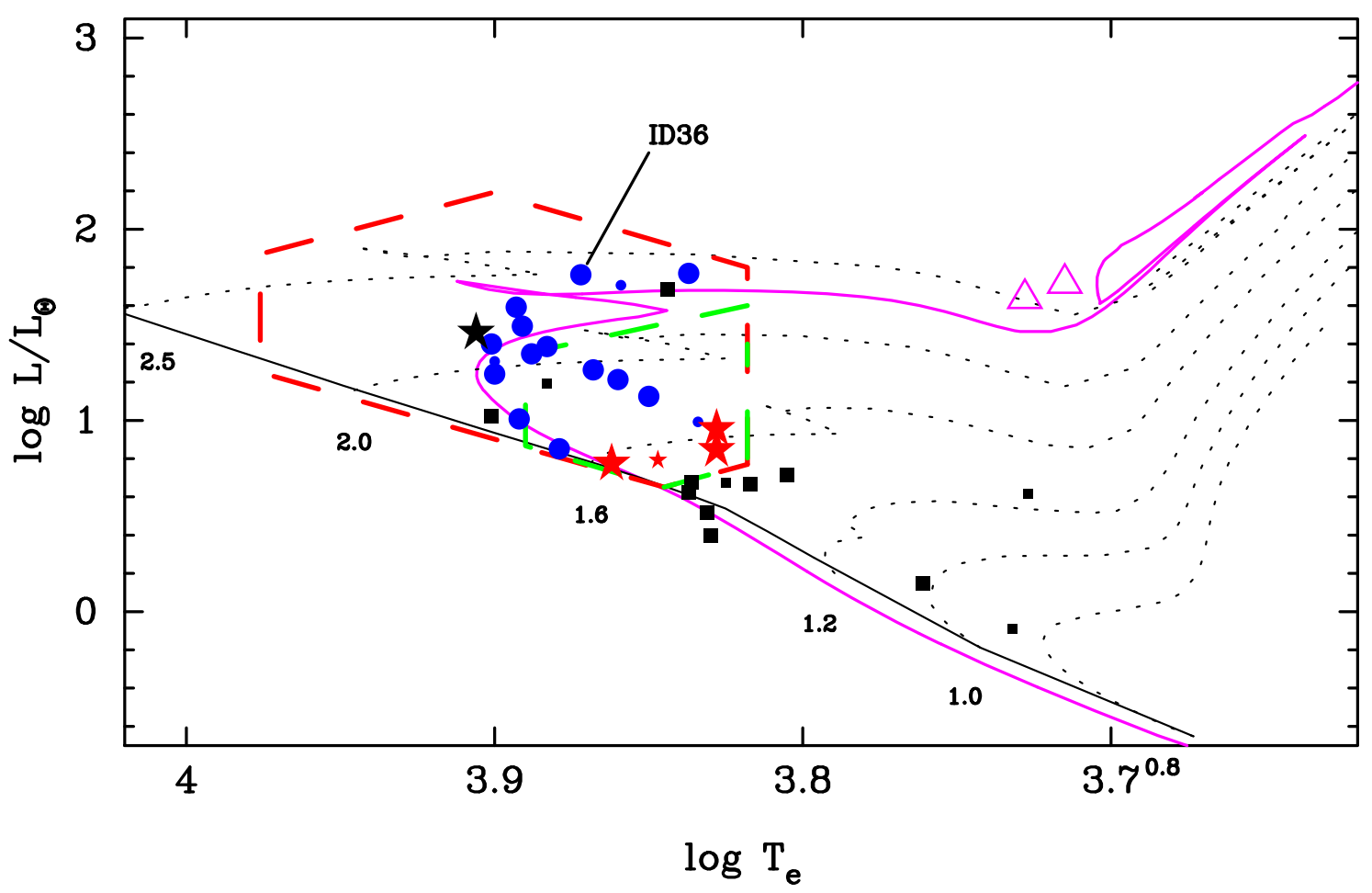

Figure 1. Hertzsprung-Russell (H-R) diagram for the star sample in NGC 6811. The zero-age main sequence (ZAMS) line and evolutionary tracks with indicated solar masses are from Claret (2004), for solar abundances. The two polygons show the general location of the Kepler $\delta$ Sct pulsators (larger polygon) and $\gamma$ Dor stars (Balona 2018c). The Padova stellar isochrone (Marigo et al. 2017) for $1 \mathrm{Gyr}, Z=0.012$, is drawn as the pink solid line. $\delta$ Sct stars in NGC 6811 are shown as blue filled circles, $\gamma$ Dor stars as red filled stars (ID34 in black), red giants with solar-like oscillations are pink triangles. The remaining stars, all showing rotational modulation, are black squares. Small symbols are non-members.

(period [d], amplitude [mmag]): ID01 (2.2, 0.1), ID15 (11.7, 1.0), ID16 (1.8, 0.1), ID23 (0.98, 0.02), and ID30 (2.3, 0.04).

Two of the new rotational variables, ID01 and ID15, are shown in Fig. 2 using one-quarter in LC mode to better illustrate the variability. Fig. 3 shows the light curves and periodograms corresponding to ID04 and ID10, two red giants with solar-like oscillations. Figs 4 and 5 present the periodograms and samples of the LC light curves of ID06 and ID07. Both of these cool stars are probably non-members of the cluster that show rotational modulation and some flaring events. Fig. 6 shows the light curves and periodograms for the $\delta$ Sct and $\gamma$ Dor stars in our sample.

An additional frequency analysis in much more depth was carried out using the SIGSPEC program (Reegen 2007), as discussed in the following sections. Results from this analysis are summarized in column 12 of Table 1 . As shown, all the $\delta$ Sct stars in the sample are essentially $\delta \mathrm{Sct} / \gamma$ Dor pulsators confirming the results by Balona (2018c). We keep the term $\delta \mathrm{Sct} / \gamma$ Dor for these stars in Table 1 to indicate the different variability types in the same order as the amplitudes, of the peaks of different origin, decrease. It is also remarkable that many of the $\delta$ Sct stars show low-frequency peaks suggestive of rotational modulation. Some examples are shown in the right-hand panels of Fig. 7, where the significant peaks in the low-frequency region are due to both $\gamma$ Dor-type pulsations and stellar rotation. The left-hand panels in Fig. 7 show three out the five $\gamma$ Dor stars in the sample.

Our results indicate that all the main-sequence non-pulsating stars in the sample show what appears to be periodic light variations consistent with the expected rotation frequency, even in the hottest stars. In summary, the sample consists of two red giants with solarlike oscillations, $16 \delta$ Sct stars, five $\gamma$ Dor stars, and 13 rotational variables.

\section{ANALYSIS OF THE MAIN-SEQUENCE PULSATORS}

Each monthly quarter Qi.j from the SC time series was analysed individually using SIGSPEC for each of the $\delta$ Sct and $\gamma$ Dor stars in the sample. SigSPEC searches for significant peaks in the periodogram iteratively until the default limit for the spectral significance (taken as SIG $=5.0$ ) is reached. A SIGSPEC limit of SIG $=5.46$ is approximately equivalent to a signal-to-noise ratio $(\mathrm{S} / \mathrm{N})=4.0$ using PERIOD04 (Reegen 2007; Kallinger, Reegen \& Weiss 2008).

When the periodogram has many closely spaced peaks, precautions need to be taken to avoid false identifications. This is due to the fact that as each frequency peak is extracted, the residual may not be entirely zero because of unavoidable errors in the frequency. These fictitious low-amplitude peaks are eventually treated as real frequencies (Balona 2014b). One way of avoiding this problem is to be much more conservative concerning the limit used for the SIG parameter (see e.g. García Hernández et al. 2009, 2013; Chapellier et al. 2011). In these cases, either a conservative significance threshold of SIG $=50$ was adopted or a maximum of 100 significant peaks was extracted.

The analysis was carried out in the frequency range $0.1-100 \mathrm{~d}^{-1}$ that covers the known frequency range of the $\delta$ Sct variables. The lower limit of $0.1 \mathrm{~d}^{-1}$ was chosen to avoid possible longterm variability produced by instrumental effects. Nevertheless, this region was also checked by visual inspection using PERIOD04 looking for any remaining peak of interest.

Finally, we confined the frequency extraction to 20 highest amplitude peaks in each star. Peaks that consisted of close pairs with a frequency separation less than the Rayleigh limit of $1 / \Delta T$ 
Table 1. Kepler stars observed in SC mode within a distance $d<7.0$ arcmin from the centre of NGC 6811 . Four additional stars from a secondary field B are included (see text for details). ID, KIC, and WEB: identification number in this work, Kepler Input Catalogue (KIC), and wEBDA data base, respectively; $d$ : the distance (arcmin) from the cluster centre; $N_{\mathrm{m}}$ : number of months with Kepler SC observations; Var. type and Source: variability types and sources from the literature: Vx (Lu09 identification), S-L (solar-like oscillations), Rot (rotational variable), EB (eclipsing binary), Bin (binary), SB1 (single-line spectroscopic binary), F (flares); effective temperatures are from Ma17; luminosities are from Gaia parallaxes or else from the KIC radius (Ma17); Mem: cluster membership (M: member; N: non-member) based on Gaia proper motions and parallaxes; This work: variability type derived in this work; Amp. var.: amplitude variability detected in this work for the main pulsation modes. The sources are: 1 - Lu09; 2 - Uy11; 3 - Mo14; 4 - Pr11; 5 - Ba13; 6 - Ba14; 7 - Ba15; 8 - Mc14; 9 Ni13; 10 - Re13; $11-\operatorname{Re} 15 ; 12-$ Me11; 13 - St11; 14 - De11; 15 - Sa16. (a) $v_{\text {rot }}$ has significance sig $>50$, but it is not among the 20 main significant peaks.

\begin{tabular}{|c|c|c|c|c|c|c|c|c|c|c|c|c|}
\hline ID & $\mathrm{KIC}$ & $\mathrm{WEB}^{a}$ & $\begin{array}{c}K_{\mathrm{p}} \\
(\mathrm{mag})\end{array}$ & $\begin{array}{c}d \\
(\operatorname{arcmin})\end{array}$ & $N_{\mathrm{m}}$ & Var. type & Source & $\log T_{\text {eff }}$ & $\log L / \mathrm{L}_{\odot}$ & Mem & This work & $\begin{array}{l}\text { Amp. } \\
\text { var. }\end{array}$ \\
\hline 01 & 9594739 & 231 & 13.95 & $8.7(\mathrm{~B})$ & 1 & & & 3.831 & 0.520 & M & Rot & \\
\hline 02 & 9594857 & 489 & 11.02 & 11.5(B) & 14 & $\begin{array}{c}\delta \mathrm{Sct}(\mathrm{V} 6) / \\
\mathrm{SB} 1 ?\end{array}$ & 1,3 & 3.837 & 1.769 & M & $\delta$ Sct $/ \gamma$ Dor & Yes \\
\hline 03 & 9655055 & 26 & 11.41 & 4.3 & 1 & $\delta$ Sct & 2 & 3.893 & 1.592 & M & $\delta$ Sct $/ \gamma$ Dor & \\
\hline 04 & 9655101 & 24 & 10.98 & 3.5 & 1 & S-L/SB1? & 3,13 & 3.715 & 1.717 & M & S-L & \\
\hline 05 & 9655114 & 18 & 12.07 & 4.4 & 15 & $\begin{array}{c}\delta \mathrm{Sct}(\mathrm{V} 1) / \\
\operatorname{Rot}\end{array}$ & $1,2,5$ & 3.868 & 1.264 & M & $\begin{array}{c}\delta \mathrm{Sct} / \gamma \text { Dor } / \\
\operatorname{Rot}^{a}\end{array}$ & Yes \\
\hline 06 & 9655129 & 23 & 13.81 & 3.1 & 6 & EB/Rot & 4,7 & 3.727 & 0.614 & $\mathrm{~N}$ & EB/Rot/F & \\
\hline 07 & 9655134 & 17 & 13.63 & 4.1 & 1 & Rot & 7,8 & 3.732 & -0.091 & $\mathrm{~N}$ & Rot/F & \\
\hline 08 & 9655151 & 31 & 13.24 & 2.7 & 1 & $\gamma$ Dor/Rot & 2,10 & 3.862 & 0.773 & M & $\gamma$ Dor & \\
\hline 09 & 9655155 & 16 & 12.15 & 3.9 & 1 & Rot & 5,9 & 3.883 & 1.193 & $\mathrm{~N}$ & Rot & \\
\hline 10 & 9655167 & 32 & 11.06 & 2.5 & 1 & S-L/SB1 & 3,13 & 3.728 & 1.638 & M & S-L & \\
\hline 11 & 9655177 & 70 & 10.85 & 4.5 & 7 & $\delta \operatorname{Sct}(\mathrm{V} 3)$ & 1,2 & 3.859 & 1.707 & $\mathrm{~N}$ & $\begin{array}{c}\delta \text { Sct } / \gamma \text { Dor } / \\
\text { Rot }\end{array}$ & Yes \\
\hline 12 & 9655288 & 4 & 12.94 & 0.8 & 7 & $\delta \operatorname{Sct}(\mathrm{V} 12)$ & 1 & 3.879 & 0.852 & M & $\delta$ Sct $/ \gamma$ Dor & Yes \\
\hline 13 & 9655306 & 60 & 13.63 & 4.9 & 1 & Rot & 8,11 & 3.817 & 0.666 & M & Rot & \\
\hline 14 & 9655346 & 5 & 12.21 & 0.1 & 1 & $\mathrm{~EB} / \delta \mathrm{Sct}$ & 15 & 3.901 & 1.400 & M & $\begin{array}{l}\mathrm{EB} / \delta \mathrm{Sct} / \\
\mathrm{Rot} / \gamma \text { Dor }\end{array}$ & \\
\hline 15 & 9655355 & 35 & 13.92 & 0.4 & 1 & & & 3.805 & 0.715 & & Rot & \\
\hline 16 & 9655357 & 57 & 13.78 & 4.2 & 1 & & & 3.830 & 0.396 & & Rot & \\
\hline 17 & 9655393 & 53 & 12.57 & 3.5 & 7 & $\begin{array}{c}\delta \operatorname{Sct}(\mathrm{V} 10) / \\
\text { Rot }\end{array}$ & $1,2,5$ & 3.892 & 1.008 & M & $\begin{array}{c}\delta \text { Sct } / \gamma \text { Dor } / \\
\operatorname{Rot}^{a}\end{array}$ & Yes \\
\hline 18 & 9655407 & 43 & 12.36 & 1.1 & 1 & $\delta \mathrm{Sct}$ & 14,15 & 3.850 & 1.125 & M & $\begin{array}{c}\delta \text { Sct/Rot/ } \\
\gamma \text { Dor }\end{array}$ & \\
\hline 19 & 9655419 & 42 & 12.66 & 1.2 & 7 & $\delta \operatorname{Sct}(\mathrm{V} 14)$ & 1,6 & 3.834 & 0.992 & $\mathrm{~N}$ & $\begin{array}{c}\delta \text { Sct/Rot/ } \\
\gamma \text { Dor }\end{array}$ & Yes? \\
\hline 20 & 9655422 & 39 & 11.50 & 1.3 & 7 & $\delta \operatorname{Sct}(\mathrm{V} 4)$ & 1,2 & 3.891 & 1.493 & M & $\delta \mathrm{Sct} / \gamma$ Dor & Yes \\
\hline 21 & 9655438 & 54 & 12.26 & 4.0 & 1 & $\delta \mathrm{Sct} / \gamma$ Dor & 2,3 & 3.860 & 1.213 & M & $\begin{array}{c}\delta \text { Sct/Rot/ } \\
\gamma \text { Dor }\end{array}$ & \\
\hline 22 & 9655458 & 48 & 14.82 & 2.2 & 1 & Rot & 8 & 3.761 & 0.148 & M & Rot & \\
\hline 23 & 9655461 & 45 & 12.04 & 1.8 & 1 & & & 3.901 & 1.023 & & Rot & \\
\hline 24 & 9655470 & 44 & 11.78 & 1.9 & 7 & $\delta \operatorname{Sct}(\mathrm{V} 11)$ & 1 & 3.888 & 1.349 & M & $\delta$ Sct $/ \gamma$ Dor & Yes \\
\hline 25 & 9655471 & 47 & 13.48 & 2.1 & 1 & Rot & 8 & 3.825 & 0.673 & $\mathrm{~N}$ & Rot & \\
\hline 26 & 9655487 & 46 & 13.15 & 2.2 & 1 & Rot & 2 & 3.828 & 0.845 & M & Rot $/ \gamma$ Dor & \\
\hline 27 & 9655501 & 49 & 12.44 & 3.0 & 1 & $\delta$ Sct $/ \gamma$ Dor & 2 & 3.900 & 1.309 & $\mathrm{~N}$ & $\delta$ Sct $/ \gamma$ Dor & \\
\hline 28 & 9655514 & 113 & 11.50 & 4.8 & 19 & $\delta \operatorname{Sct}(\mathrm{V} 5)$ & $1,2,3$ & 3.883 & 1.386 & M & $\begin{array}{c}\delta \text { Sct } / \gamma \text { Dor } / \\
\operatorname{Rot}^{a}\end{array}$ & Yes \\
\hline 29 & 9655543 & 106 & 11.37 & 3.9 & 1 & Rot & 9 & 3.844 & 1.683 & M & Rot & \\
\hline 30 & 9655708 & 163 & 13.75 & $7.2(\mathrm{~B})$ & 1 & & & 3.837 & 0.624 & M & Rot & \\
\hline 31 & 9655800 & 230 & 12.67 & 7.9(B) & 1 & $\gamma$ Dor/Rot & 2,10 & 3.847 & 0.792 & $\mathrm{~N}$ & $\gamma$ Dor $/ \operatorname{Rot}^{a}$ & \\
\hline 32 & 9716076 & 81 & 13.57 & 4.2 & 1 & Rot & 12 & 3.836 & 0.675 & M & Rot & \\
\hline 33 & 9716220 & 33 & 11.88 & 2.6 & 7 & $\begin{array}{c}\delta \operatorname{Sct}(\mathrm{V} 13) / \\
\text { Rot }\end{array}$ & $1,3,5$ & 3.900 & 1.242 & M & $\begin{array}{c}\delta \text { Sct } / \gamma \text { Dor } / \\
\text { Rot? }\end{array}$ & Yes \\
\hline 34 & 9716301 & 34 & 11.62 & 2.2 & 1 & Rot & 5 & 3.906 & 1.458 & M & $\gamma$ Dor/Rot & \\
\hline 35 & 9716358 & 36 & 13.26 & 0.9 & 1 & & & 3.828 & 0.954 & & Rot $/ \gamma$ Dor & \\
\hline 36 & 9716385 & 37 & 10.86 & 1.5 & 19 & $\delta \operatorname{Sct}(\mathrm{V} 2)$ & 1 & 3.872 & 1.762 & M & $\begin{array}{c}\delta \mathrm{Sct} / \mathrm{Bin} / \\
\operatorname{Rot} / \gamma \operatorname{Dor} / \mathrm{F}\end{array}$ & Yes \\
\hline
\end{tabular}

${ }^{a}$ http://webda.physics.muni.cz

$\sim 0.03 \mathrm{~d}^{-1}$ ( $\Delta T$ being the total time span of the observations) were ignored. In stars with SC observations spanning many months, the resulting set of 20 frequencies derived from the first available quarter was compared with those derived from subsequent quarters, in order to check if the same frequencies were present. This was found to be the case for all stars with sufficient observations. Using the frequencies found in the first available quarter, a simultaneous least-squares solution for all 20 frequencies was performed for the whole data set. This solution was then used to investigate how the amplitudes may vary with time. 

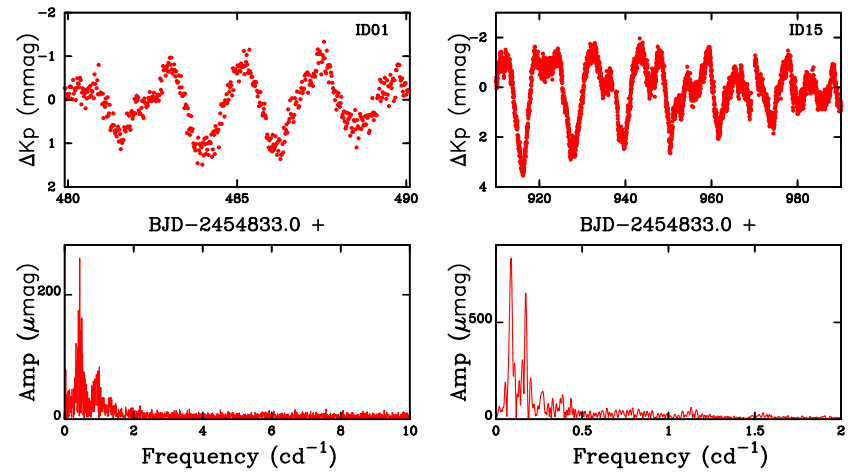

Figure 2. Two examples of stars, ID01 and ID15, showing rotational modulation. Top panels: light curves corresponding to one-quarter of longcadence (LC) observations. Bottom panels: resulting periodograms.
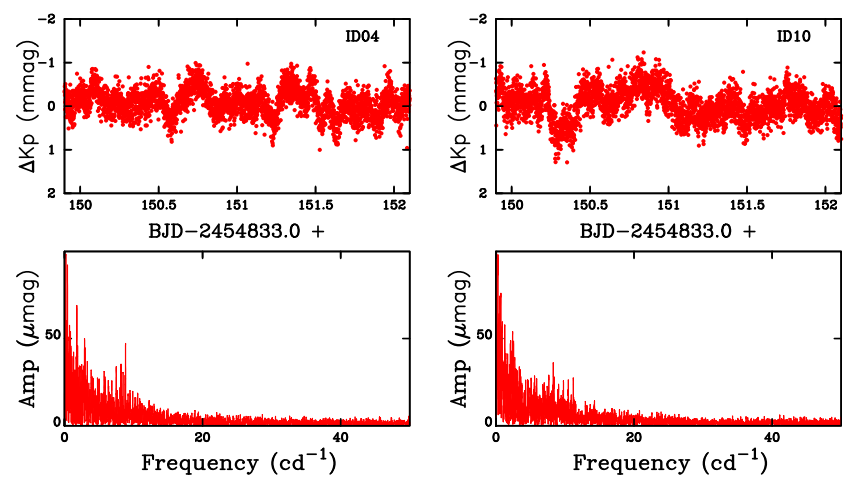

Figure 3. The two red giants in the sample, ID04 and ID10, showing solar-like oscillations. Top panels: light curves corresponding to 1 month of Kepler SC observations. Bottom panels: resulting periodograms. The increase in power towards low frequencies suggests surface activity.

It is also of interest to investigate the low-frequency region. Low frequencies are usually attributed to independent g-modes as found in $\gamma$ Dor stars. They could also be a result of Rossby waves (Saio et al. 2018), which are waves driven by the interaction between gravitation and rotation. They may also arise as a result of non-linear combinations between pairs of frequencies in the high-frequency region (the parent modes). The parent modes are likely to be of high amplitude, so this may be confirmed by looking for an appropriate combination among the extracted frequencies.

Low frequencies may also be unrelated to pulsation, such as eclipses in a binary system, tidal distortion as found in ellipsoidal variables, or stellar activity as a result of star-spots and rotational modulation. If one or more harmonics of a lowfrequency peak are present, it may possibly be a result of nonlinear pulsation, but this is also an indication of binarity or starspots. Harmonics of a pulsational mode are hard to detect unless the parent mode has a large amplitude. It is not possible to distinguish between these scenarios unless significant amplitude variation is present. If that is the case, the possibility of pulsation, binarity, and tidal effects is considerably reduced and one can reasonably conclude that the variations may be due to a starspot that varies in size or location, particularly if the amplitude is large.
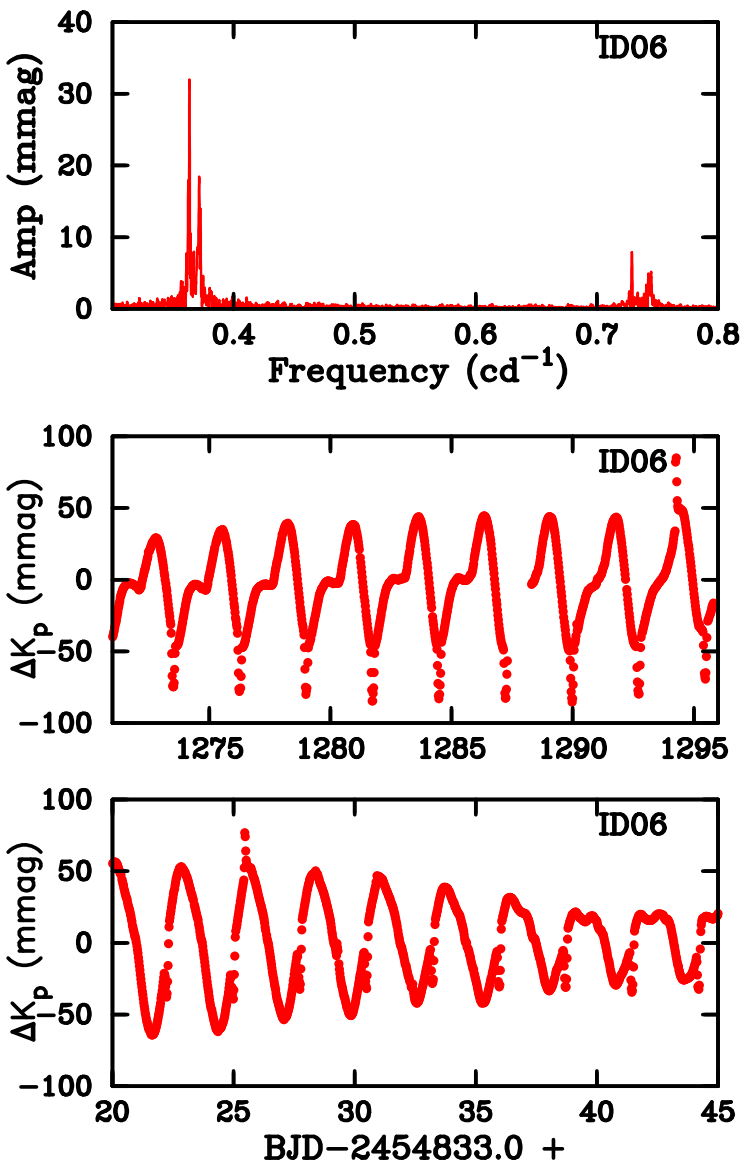

Figure 4. Top panel: periodogram of the LC data for ID06 showing two closely spaced peaks and their harmonics. The peak with larger amplitude is due to eclipses, and the other, broad peak, is due to rotation suggesting slightly asynchronous rotation. Middle and bottom panels: extracts from the LC light curve showing the rotational modulation and two flaring events.
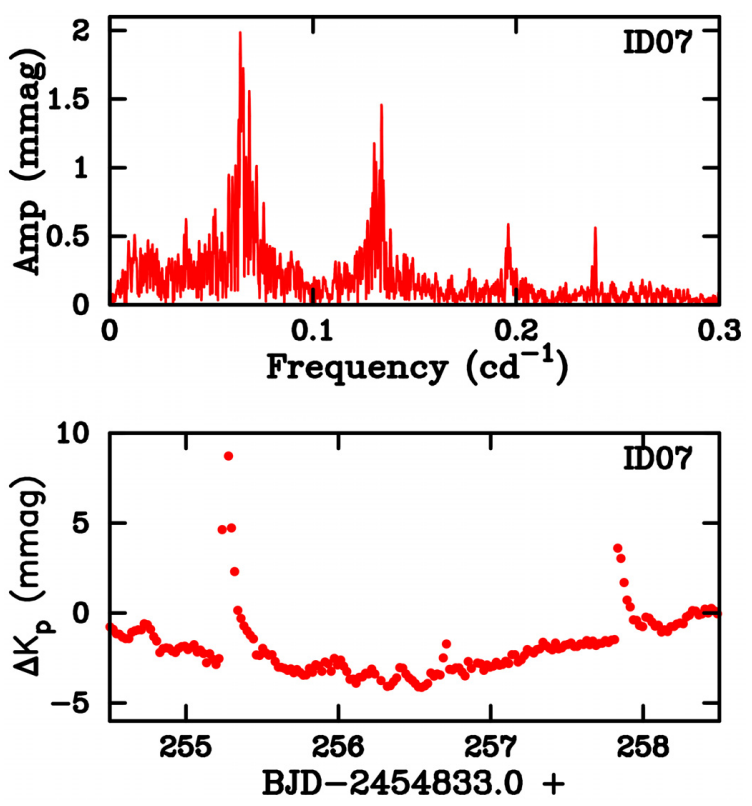

Figure 5. Top panel: periodogram of the LC data for ID07 showing the rotational peaks at $v_{\text {rot }}=0.064 \mathrm{~d}^{-1}$ and its first two harmonics. The peak at $0.239 \mathrm{~d}^{-1}$ is of unknown origin. Bottom panel: extract from the LC light curve in which two flares can be seen. 

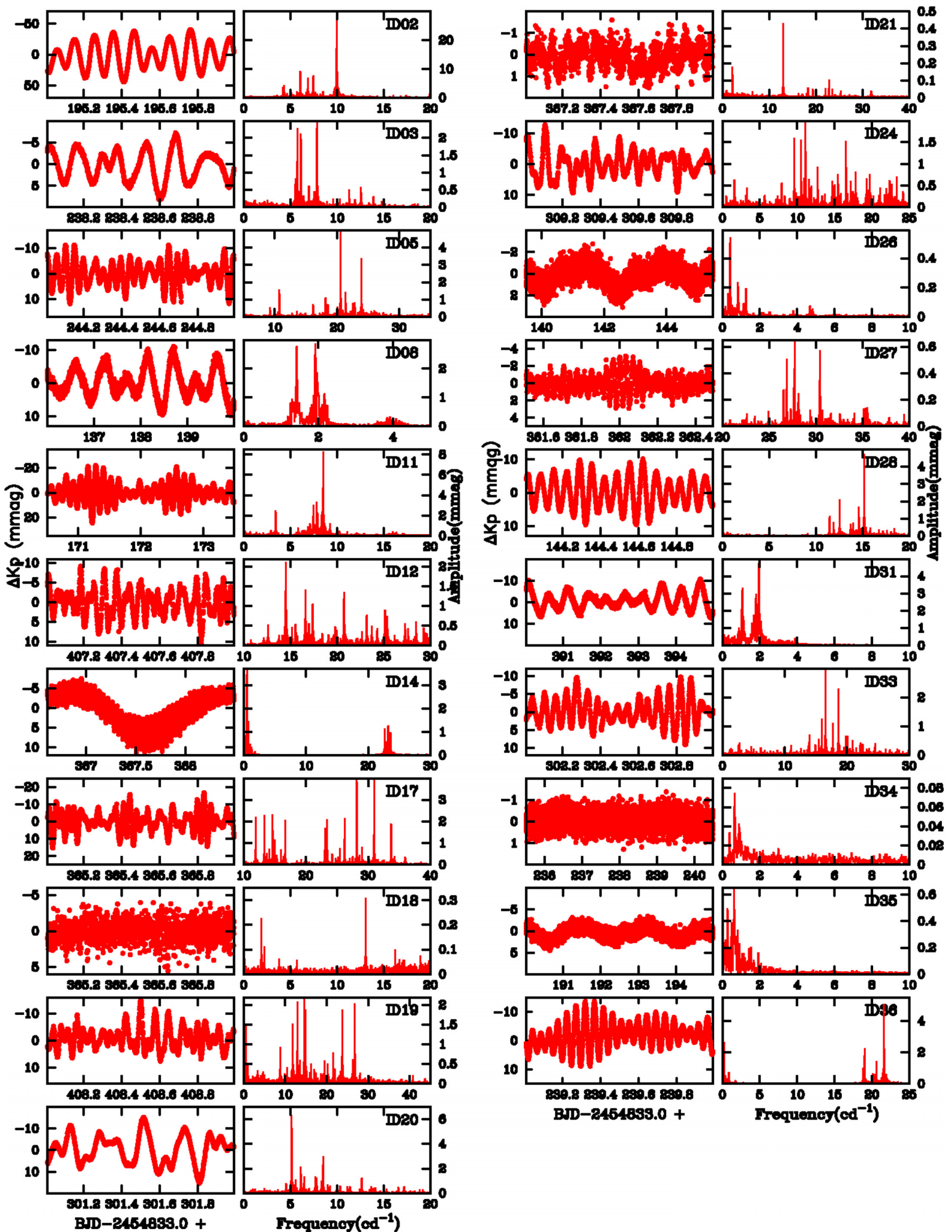

Figure 6. $\delta$ Sct and $\gamma$ Dor stars in the sample: light curves in SC mode (left-hand panels) and zooms to the periodograms in the regions of interest (right-hand panels). 

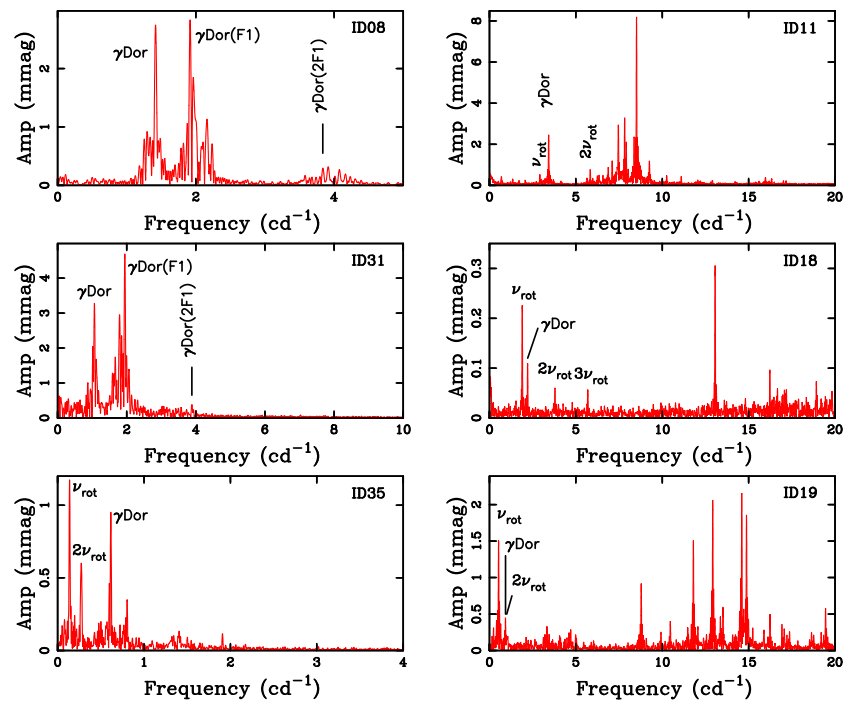

Figure 7. $\gamma$ Dor (left-hand panels) and $\delta$ Sct (right-hand panels) stars showing rotation and g-mode peaks in the low-frequency regions using SC data, except for ID35, where the LC Q5 quarter is used.

Table 2. $\delta$ Sct- and $\gamma$ Dor-type pulsators of Group I. ID: identification number; Qi.j: quarter; $\Delta T$ : time span; $N_{\mathrm{p}}$ : number of peaks detected with significance sig $>50$; Type: variability type according to the main peaks.

\begin{tabular}{lllll}
\hline ID $\quad$ Qi.j & $\Delta T$ & $N_{\mathrm{p}}$ & Type
\end{tabular}

(d)

\begin{tabular}{lcccc}
\hline 03 & 2.3 & 27 & $>100$ & $\delta$ Sct \\
08 & 1.3 & 33 & 55 & $\gamma$ Dor \\
14 & 4.1 & 31 & 26 & $\delta$ Sct \\
18 & 4.1 & 31 & 7 & $\delta$ Sct \\
21 & 4.1 & 31 & 7 & $\delta$ Sct \\
26 & 1.3 & 33 & 10 & $\gamma$ Dor \\
27 & 4.1 & 31 & 45 & $\delta$ Sct \\
31 & 4.2 & 31 & 54 & $\gamma$ Dor \\
34 & 2.3 & 27 & 4 & $\gamma$ Dor \\
35 & 2.1 & 31 & 15 & $\gamma$ Dor \\
\hline
\end{tabular}

\section{GROUP I: $\delta$ SCT AND $\gamma$ DOR STARS WITH LIMITED OBSERVATIONS}

Group I in our sample consists of 10 stars observed in SC mode but for only 1 month. They are listed in Table 2 together with the quarters, Qi.j, in which the observations were made, the time span, $\Delta T$, and the number of extracted peaks with sig $>50$ significance. Results for the 20 highest amplitude peaks are given in Table B1 where appropriate. The two last columns list the amplitude and S/N for each peak, respectively. Noise levels are determined from the residuals after pre-whitening 100 peaks from the periodogram. If the number of significant peaks is less than 100, the noise level is calculated from the corresponding residuals at that stage. Frequency identifications of interest are shown in Table 3. Frequencies that might be a result of rotational modulation are shown only if they have variable amplitudes and if the harmonic is visible, as explained above. To determine possible amplitude variability, the LC light curves were used since they cover a much longer time span.

All the stars listed in Table 2, except for ID03, have far fewer than 100 significant peaks. This is because their amplitudes are very
Table 3. Identifications of interest for the $\delta$ Sct- and $\gamma$ Dor-type stars of Group I. Fx: peaks with significance sig $<50$ or from the full LC time series.

\begin{tabular}{|c|c|c|c|c|}
\hline $\begin{array}{l}\text { Iden } \\
\left(\mathrm{d}^{-1}\right)\end{array}$ & $\mathrm{Fi}$ & $\begin{array}{l}\text { Freq } \\
\left(d^{-1}\right)\end{array}$ & $\begin{array}{c}\text { Amp } \\
(\mathrm{mmag})\end{array}$ & Sig \\
\hline \multicolumn{5}{|l|}{ ID03 (Q2.3) } \\
\hline $2 \mathrm{~F} 4=11.0918$ & $\mathrm{Fx}(\mathrm{LC})$ & 11.0914 & 0.020 & - \\
\hline $\mathrm{F} 1+\mathrm{F} 4=13.3888$ & F93 & 13.3850 & 0.058 & 67 \\
\hline $\mathrm{F} 2+\mathrm{F} 4=11.2972$ & F7 & 11.2935 & 0.497 & 1064 \\
\hline $\mathrm{F} 2-\mathrm{F} 4=0.2054$ & F17 & 0.2064 & 0.175 & 218 \\
\hline $\mathrm{F} 3+\mathrm{F} 4=11.6639$ & F61 & 11.6709 & 0.081 & 95 \\
\hline $\mathrm{F} 3-\mathrm{F} 4=0.5721$ & F48 & 0.5634 & 0.098 & 118 \\
\hline \multicolumn{5}{|l|}{ ID08 (Q1.3) } \\
\hline $\mathrm{F} 1=1.9221(\mathrm{LC})$ & $\mathrm{F} 1$ & 1.9174 & 2.781 & 3185 \\
\hline $2 \mathrm{~F} 1=3.8442$ & $\mathrm{Fx}(\mathrm{LC})$ & 3.8442 & 0.173 & - \\
\hline $3 \mathrm{~F} 1=5.7663$ & $\mathrm{Fx}(\mathrm{LC})$ & 5.7662 & 0.014 & - \\
\hline $\mathrm{F} 2=1.4155(\mathrm{LC})$ & $\mathrm{F} 2$ & 1.4170 & 2.670 & 4164 \\
\hline $2 \mathrm{~F} 2=3.8310$ & $\mathrm{Fx}(\mathrm{LC})$ & 3.8311 & 0.053 & - \\
\hline $\mathrm{F} 1-\mathrm{F} 2=0.5066$ & F47 & 0.5065 & 0.097 & 73 \\
\hline $\mathrm{F} 1+\mathrm{F} 2=3.3376$ & Fx & 3.339 & 0.050 & $<50$ \\
\hline $\mathrm{F} 1+\mathrm{F} 3=3.9057$ & F54 & 3.8970 & 0.080 & 53 \\
\hline $\mathrm{F} 1+\mathrm{F} 4=4.0829$ & $\mathrm{~F} 23$ & 4.0782 & 0.244 & 327 \\
\hline $\mathrm{F} 1-\mathrm{F} 4=0.2387$ & Fx & 0.241 & 0.080 & $<50$ \\
\hline \multicolumn{5}{|l|}{ ID14 (Q4.1) } \\
\hline$v_{\mathrm{orb}}=0.2568(\mathrm{LC})$ & $\mathrm{Fx}(\mathrm{LC})$ & 0.2568 & 0.440 & - \\
\hline$v_{\text {rot }}=0.2720(\mathrm{LC})$ & F6 & 0.2772 & 0.961 & 2410 \\
\hline $2 v_{\mathrm{orb}}=0.5136$ & $\mathrm{~F} 1$ & 0.5141 & 3.637 & 5888 \\
\hline $3 v_{\text {orb }}=0.7704$ & $\mathrm{~F} 2$ & 0.7696 & 1.448 & 2298 \\
\hline $4 v_{\text {orb }}=1.0272$ & F9 & 1.0266 & 0.454 & 1317 \\
\hline $5 v_{\text {orb }}=1.2840$ & F11 & 1.2847 & 0.319 & 854 \\
\hline $7 v_{\text {orb }}=1.7976$ & F16 & 1.7968 & 0.156 & 271 \\
\hline $2 v_{\text {rot }}=0.5440$ & F10 & 0.5447 & 0.387 & 1141 \\
\hline \multicolumn{5}{|l|}{ ID18 (Q4.1) } \\
\hline \multicolumn{5}{|l|}{$\mathrm{F} 2=1.8937=v_{\mathrm{rot}}$} \\
\hline $2 \mathrm{~F} 2=3.7874$ & F6 & 3.7881 & 0.060 & $<50$ \\
\hline $3 \mathrm{~F} 2=5.6811$ & F7 & 5.6880 & 0.056 & $<50$ \\
\hline \multicolumn{5}{|l|}{ ID21 (Q4.1) } \\
\hline$v_{\mathrm{rot}}=2.1231(\mathrm{LC})$ & $\mathrm{F} 2$ & 2.1239 & 0.181 & 800 \\
\hline $2 v_{\mathrm{rot}}=4.2462$ & $\operatorname{Fx}(\mathrm{LC})$ & 4.2464 & 0.039 & - \\
\hline $3 v_{\text {rot }}=6.3693$ & $\mathrm{Fx}(\mathrm{LC})$ & 6.3690 & 0.028 & - \\
\hline $4 v_{\text {rot }}=8.4924$ & $\operatorname{Fx}(\mathrm{LC})$ & 8.4920 & 0.006 & - \\
\hline \multicolumn{5}{|l|}{ ID26 (Q1.3) } \\
\hline$v_{\text {rot }}=0.4065(\mathrm{LC})$ & $\mathrm{F} 1$ & 0.4088 & 0.529 & 2869 \\
\hline $2 v_{\text {rot }}=0.8130$ & $\mathrm{~F} 2$ & 0.8233 & 0.225 & 730 \\
\hline $3 v_{\text {rot }}=1.2195$ & F9 & 1.2139 & 0.073 & 80 \\
\hline \multicolumn{5}{|l|}{ ID31 (Q4.2) } \\
\hline $\mathrm{F} 1=1.9405(\mathrm{LC})$ & $\mathrm{F} 1$ & 1.9445 & 4.781 & 2944 \\
\hline $2 \mathrm{~F} 1=3.8810$ & $\mathrm{Fx}(\mathrm{LC})$ & 3.8811 & 0.263 & - \\
\hline $3 \mathrm{~F} 1=5.8216$ & $\operatorname{Fx}(\mathrm{LC})$ & 5.8216 & 0.017 & - \\
\hline$v_{\mathrm{rot}}=0.1513(\mathrm{LC})$ & $\operatorname{Fx}(\mathrm{LC})$ & 0.1513 & 0.366 & - \\
\hline $2 v_{\mathrm{rot}}=0.3026$ & $\mathrm{Fx}(\mathrm{LC})$ & 0.3026 & 0.167 & - \\
\hline $\mathrm{F} 1-\mathrm{F} 2=0.8756$ & F13 & 0.8767 & 0.528 & 743 \\
\hline $\mathrm{F} 1+\mathrm{F} 3=3.7320$ & F29 & 3.7324 & 0.239 & 275 \\
\hline \multicolumn{5}{|l|}{ ID34 (Q2.3) } \\
\hline$v_{\text {rot }}=0.5014(\mathrm{LC})$ & $\mathrm{Fx}(\mathrm{LC})$ & 0.5014 & 0.014 & - \\
\hline $2 v_{\mathrm{rot}}=1.0028$ & $\mathrm{Fx}(\mathrm{LC})$ & 1.0026 & 0.009 & - \\
\hline $3 v_{\text {rot }}=1.5042$ & $\mathrm{Fx}(\mathrm{LC})$ & 1.5037 & 0.0042 & - \\
\hline $4 v_{\text {rot }}=2.0056$ & $\operatorname{Fx}(\mathrm{LC})$ & 2.0048 & 0.0038 & - \\
\hline \multicolumn{5}{|l|}{ ID35 (Q2.1) } \\
\hline$v_{\text {rot }}=0.1351(\mathrm{LC})$ & F7 & 0.1380 & 0.253 & 335 \\
\hline $2 v_{\mathrm{rot}}=0.2702$ & $\operatorname{Fx}(\mathrm{LC})$ & 0.2715 & 0.160 & - \\
\hline $3 v_{\text {rot }}=0.4053$ & $\mathrm{Fx}(\mathrm{LC})$ & 0.4065 & 0.060 & - \\
\hline $\mathrm{F} 1-v_{\mathrm{rot}}=0.4835$ & $\operatorname{Fx}(\mathrm{LC})$ & 0.4766 & 0.109 & - \\
\hline $\mathrm{F} 1+v_{\text {rot }}=0.7537$ & $\operatorname{Fx}(\mathrm{LC})$ & 0.7539 & 0.102 & - \\
\hline
\end{tabular}


small. For example, the highest peak in ID18, ID21, and ID27 has an amplitude in the range $0.3-0.6$ mmag. For ID14, the main pulsation peak is F3 with amplitude $1.2 \mathrm{mmag}$. The $\gamma$ Dor stars have less complex power spectra than $\delta$ Sct stars with similar amplitudes.

Two of the $\gamma$ Dor pulsators, ID08 and ID31, were already classified as $\gamma$ Dor pulsators by Uytterhoeven et al. (2011) or as rotational variables by Reinhold et al. (2013). For ID08, Reinhold et al. (2013) listed two possible rotation periods, $P_{1}=0.5203 \mathrm{~d}$ and $P_{2}=0.7067 \mathrm{~d}$, which correspond to F1 and F2, our two largest amplitude frequencies. However, although using the LC data we can identify their harmonics $2 \mathrm{~F} 1$ and $3 \mathrm{~F} 1$, or $2 \mathrm{~F} 2$ (see Table 3), these peaks are likely produced by g-mode pulsations. Their sharp appearance and nearly stable temporal evolution of the amplitudes, together with the very small amplitude ratio values of $A(2 \mathrm{~F} 1) / A(\mathrm{~F} 1)$, $A(3 \mathrm{~F} 1) / A(2 \mathrm{~F} 1)$, and $A(2 \mathrm{~F} 2) / A(\mathrm{~F} 2)$ (only a few per cent), indicate that these peaks have a pulsation origin.

In a similar way, although Reinhold et al. (2013) listed two rotation periods for ID31 corresponding to F1 and F3 in our Table B1, we find that they are produced by $\gamma$ Dor pulsations. Nevertheless, the harmonic of $v=0.1513 \mathrm{~d}^{-1}$ is present (Table 3), and this may possibly be identified with the rotational frequency. Interestingly, in both ID08 and ID31 there are similar sequences of peaks of almost equal separation that deserve closer investigation. It may be a sequence of dipole gravity g-modes as seen in KIC 5123889 (Balona 2018a) and other stars.

ID35 is a new $\gamma$ Dor variable with very small amplitude. The analysis of the SC data suggests $\mathrm{F} 1=0.6186 \mathrm{~d}^{-1}$ as a $\gamma$ Dor pulsation peak and $\mathrm{F} 7=0.1380 \mathrm{~d}^{-1}$ as a rotational peak. This is supported by an analysis of the full 4-yr LC time series, as shown in Table 3. Moreover, the rotational peak is the one with the largest amplitude during some LC quarters, as shown in Fig. 7 for quarter Q5. Furthermore, none of these three $\gamma$ Dor stars show any significant peak in the high-frequency domain $\left(v>5 \mathrm{~d}^{-1}\right)$, which is confirmed when the corresponding LC time series are analysed.

ID14 has recently been detected as an eclipsing binary with a $\delta$ Sct component (Sandquist et al. 2016). Our analysis confirms this result and reveals some additional peaks in the low-frequency domain as result of rotation and $\gamma$ Dor-type pulsations. Extracts of the periodogram of the LC data are shown in Fig. 8 showing the orbital frequency at $v_{\text {orb }}=0.2568 \mathrm{~d}^{-1}$ and the rotation frequency at $v_{\text {rot }}=$ $0.2720 \mathrm{~d}^{-1}$ suggesting slightly asynchronous rotation similar to ID06 (Fig. 4). The periodogram in the high-frequency region shows a set of peaks in the very narrow $22-24 \mathrm{~d}^{-1}$ frequency range, which is due to $\delta$ Sct pulsations. Although the frequency distributions in $\delta$ Sct stars vary tremendously from one object to another, it is very unusual that the peaks are confined to such a narrow range.

ID18 and ID21 are two $\delta$ Sct stars with very low amplitudes. ID21 was classified as a 'hybrid' $\delta$ Sct $/ \gamma$ Dor variable by Uytterhoeven et al. (2011). The hybrid character of ID18 is confirmed by our analysis (F3 peak) together with rotation (F2 peak). Both characteristics are also confirmed for ID21 when the LC time series is used. One strong peak is present at $v=2.1231 \mathrm{~d}^{-1}$ together with its first three harmonics (Table 3). The broad appearance and temporal evolution of this peak over the $4 \mathrm{yr}$ of LC data, with significant variations in amplitude, indicate that it can be identified as a rotational peak.

Uytterhoeven et al. (2011) also list ID27 as a 'hybrid' $\delta$ Sct $/ \gamma$ Dor star with very low amplitude. The periodogram is dominated by peaks in the frequency range $26-32 \mathrm{~d}^{-1}$. No rotational peak seems to be present, but there is a low-frequency mode F9 $=1.7286 \mathrm{~d}^{-1}$ with relatively high amplitude in addition to a number of low frequencies with lower amplitudes.
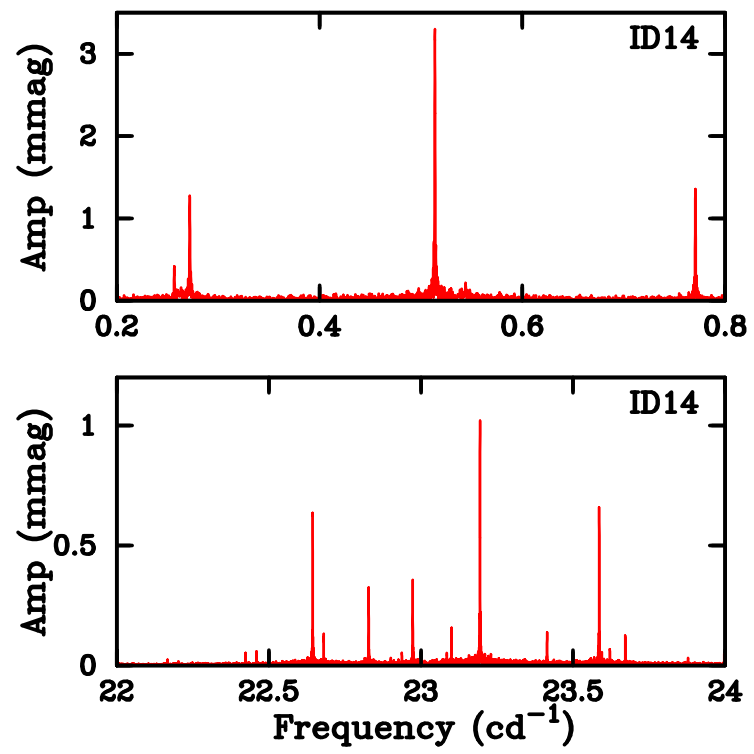

Figure 8. Periodograms of the LC data for ID14. Top panel: low-frequency region showing the orbital frequency at $v_{\text {orb }}=0.2568 \mathrm{~d}^{-1}$ and its first two harmonics, and another set of peaks with the rotational frequency at $v_{\text {rot }}=$ $0.2720 \mathrm{~d}^{-1}$ and its first harmonic suggesting slightly asynchronous rotation. Bottom panel: high-frequency region showing a set of peaks in the very narrow range $22-24 \mathrm{~d}^{-1}$ that are due to $\delta$ Sct pulsations.

The largest amplitude $\delta$ Sct star in Group I is ID03 (Uytterhoeven et al. 2011). Fig. 6 and Table B 1 show that the variability is dominated by three peaks in the range $5.5-8 \mathrm{~d}^{-1}$ with amplitudes around 2.0-2.5 mmag. A number of low-frequency peaks are also present (F14, F17, F18, and F19). F17 can be identified as the combination $\mathrm{F} 17=\mathrm{F} 2-\mathrm{F} 4$, while the others can be interpreted as $\gamma$ Dor g-modes. The first harmonic of F4 and some combination frequencies in which the three main peaks are parent modes are also listed in Table 3. The LC data are useful in confirming these identifications. F4 also shows significant amplitude variation in the full LC time series, but the amplitude seems rather large for rotational modulation. Furthermore, the amplitude ratio $A_{2 \mathrm{~F} 4} / A_{\mathrm{F} 4}=$ 0.027 is quite small.

There are two additional stars in Group I, ID26 and ID34, which also appear as $\gamma$ Dor variables with rotational modulation. The periodogram of ID26 is dominated by a broad rotational peak at $v_{\text {rot }}=0.41 \mathrm{~d}^{-1}$ and its first two harmonics (Tables B1 and 3). In addition, a series of sharp peaks with frequencies up to $5 \mathrm{~d}^{-1}$ identify this star as a $\gamma$ Dor variable.

ID34 seems to be one of the hot $\gamma$ Dor stars discussed in Balona et al. (2016). This is the target with the lowest amplitude photometric periodicities in our sample. Only four peaks appear as significant in the periodograms of the SC data. When the LC data are used, the frequency spectrum does not resemble a typical $\gamma$ Dor star, but no other classification is consistent with the low-frequency spectrum of this star. The presumed pulsation peaks are all broad and unresolved, even in the full 4-yr LC data. On the other hand, one peak is clearly revealed at $v=0.5014 \mathrm{~d}^{-1}$ together with its first three harmonics, which are due to rotation.

\section{GROUP II: $\delta$ SCT STARS WITH 7 MONTHS OF SC OBSERVATIONS}

Group II consists of seven $\delta$ Sct stars, each one observed for 7 months in SC mode. They are listed in Table 4, which shows 
Table 4. $\delta$ Sct stars of Group II. ID: identification number; Lu09: identification number by Lu09; Qi.j: quarter; $N_{\mathrm{p}}$ : number of peaks detected with significance sig $>50$.

\begin{tabular}{cccc}
\hline ID & Lu09 & Qi.j & $N_{\mathrm{p}}$ \\
\hline 11 & V3 & $2.1,5,6$ & $>100$ \\
12 & V12 & $4.2,5,6$ & $>100$ \\
17 & V10 & $4.1,5,6$ & $\sim 90$ \\
19 & V14 & $4.2,5,6$ & $>100$ \\
20 & V4 & $3.2,5,6$ & $>100$ \\
24 & V11 & $3.2,5,6$ & $>100$ \\
33 & V13 & $3.2,5,6$ & $>100$ \\
\hline
\end{tabular}

the Qi.j quarters when they were observed and the number of peaks with significance sig $>50$. All stars have been classified as $\delta$ Sct variables by Luo et al. (2009). ID11 and ID20 (V3 and V4 in Luo et al. 2009) had already been classified as such also by van Cauteren et al. (2005). Except for ID17, the number of significant peaks is larger than 100. Table B2 shows the results for the 20 principal peaks and the first SC quarter in which observations were made. In all cases, the set of 20 highest amplitude peaks was fitted as a function of time to determine the amplitude and frequency stability of the peaks. Frequency identifications of interest are shown in Table 5. When necessary, the LC data were used as a cross-check.

The frequency spectrum of ID11 is dominated by the main peak at around $8.5 \mathrm{~d}^{-1}$, which is also the peak of largest amplitude among the variables in Group II. There appear to be frequency combinations in which the three main peaks are parent modes, as shown in Table 5. Three other peaks are in the low-frequency region: $\mathrm{F} 4=3.4230 \mathrm{~d}^{-1}$ seems to be a pulsation peak in the $\gamma$ Dor regime, whereas F17 $=2.9112 \mathrm{~d}^{-1}$ and $\mathrm{F} 12=5.8293 \mathrm{~d}^{-1}$ may be a result of rotation since $\mathrm{F} 12=2 \mathrm{~F} 17$. Evidence for this identification is confirmed by the amplitude variation of both modes as a function of time being very similar. The second harmonic, 3F17, is also detected in some SC quarters and confirmed by the LC light curve. The larger amplitude of F12 compared to F17 suggests two spots diametrically opposite each other.

In the case of ID17, the two main oscillation frequencies, F1 and F2, are beyond the LC Nyquist limit. None of the 20 main peaks fall in the low-frequency region. A few frequency combinations of very low amplitude can be detected in SC quarter Q4.1. The periodograms (see Fig. 6) show two well-defined regions 12-17 and 23$34 \mathrm{~d}^{-1}$ with similar well-defined structures. The spacing between some adjacent peaks suggests that both structures are related to each other. Balona (2013) reported $v=1.753 \mathrm{~d}^{-1}$ and its harmonic in the LC data as due to rotation. This is confirmed in our analysis of the LC data. Using the 4-yr LC data, two peaks $v_{1}=1.7535 \mathrm{~d}^{-1}$ (Amp $=0.205 \mathrm{mmag}$ ) and $v_{2}=3.5080 \mathrm{~d}^{-1}$ (Amp $\left.=0.085 \mathrm{mmag}\right)$, which can be identified as its first harmonic, are detected. The peak at $v_{1}=1.7535 \mathrm{~d}^{-1}$ really consists of two very close peaks at $v_{1}=$ 1.7535 and $v_{1}^{\prime}=1.7545 \mathrm{~d}^{-1}$. Moreover, a few independent peaks of very low amplitude with frequencies below $2 \mathrm{~d}^{-1}$ also seem to be present and indicate pulsations in the g-mode regime.

The periodogram of ID19 is dominated by pulsations in the wide range $8.5-27 \mathrm{~d}^{-1}$. However, two peaks in the low-frequency region are among the highest amplitude ones. F6 $=0.5246 \mathrm{~d}^{-1}$ is clearly due to stellar rotation, whereas F18 $=0.9240 \mathrm{~d}^{-1}$ seems to be an independent mode. For the latter, the possibility of a binary nature, with F18 as the orbital frequency, was also investigated
Table 5. Identifications of interest for the $\delta$ Sct stars of Group II. For each star, the corresponding first month Qi.j of SC observations is indicated. Parentheses show peaks coming from a different quarter. Fx: peaks with significance sig $<50$ or from the full LC time series.

\begin{tabular}{|c|c|c|c|c|}
\hline $\begin{array}{l}\text { Iden } \\
\left(d^{-1}\right)\end{array}$ & $\mathrm{Fi}$ & $\begin{array}{l}\text { Freq } \\
\left(d^{-1}\right)\end{array}$ & $\begin{array}{c}\text { Amp } \\
(\mathrm{mmag})\end{array}$ & Sig \\
\hline \multicolumn{5}{|l|}{ ID11 (Q2.1) } \\
\hline $\mathrm{F} 1+\mathrm{F} 2=15.9677$ & $\mathrm{~F} 21$ & 15.9703 & 0.492 & 297 \\
\hline $\mathrm{F} 1+\mathrm{F} 3=16.3319$ & F25 & 16.3293 & 0.345 & 241 \\
\hline $\mathrm{F} 1-\mathrm{F} 3=0.6873$ & F16 & 0.6887 & 0.480 & 346 \\
\hline $2 \mathrm{~F} 1=17.0192$ & F50 & 17.0143 & 0.197 & 117 \\
\hline \multicolumn{5}{|l|}{$\mathrm{F} 17=2.9112=v_{\text {rot }}$} \\
\hline $2 \mathrm{~F} 17=5.8224$ & F12 & 5.8293 & 0.821 & 801 \\
\hline $3 \mathrm{~F} 17=8.7336$ & F86(Q5.1) & 8.7355 & 0.129 & 126 \\
\hline \multicolumn{5}{|l|}{ ID12 (Q4.2) } \\
\hline \multirow[t]{2}{*}{$\mathrm{F} 2-\mathrm{F} 1=2.1152$} & Fx & 2.111 & 0.110 & $<50$ \\
\hline & $\mathrm{Fx}(\mathrm{Q} 5.1)$ & 2.116 & 0.115 & $<50$ \\
\hline \multirow[t]{2}{*}{$\mathrm{F} 3-\mathrm{F} 1=6.2395$} & Fx & 6.233 & 0.090 & $<50$ \\
\hline & $\mathrm{Fx}(\mathrm{Q} 5.1)$ & 6.237 & 0.080 & $<50$ \\
\hline \multicolumn{5}{|l|}{ ID17 (Q4.1) } \\
\hline$v_{\mathrm{rot}}=1.7535(\mathrm{LC})$ & F27 & 1.7454 & 0.282 & 294 \\
\hline $2 v_{\mathrm{rot}}=3.5070$ & $\operatorname{Fx}(\mathrm{LC})$ & 3.5080 & 0.085 & - \\
\hline $\mathrm{F} 1+\mathrm{F} 6=54.3682$ & $\mathrm{Fx}$ & 54.363 & 0.090 & $<50$ \\
\hline $\mathrm{F} 1+\mathrm{F} 2=59.1070$ & Fx & 59.106 & 0.070 & $<50$ \\
\hline $\mathrm{F} 1+\mathrm{F} 9=61.8416$ & $\mathrm{Fx}$ & 61.841 & 0.055 & $<50$ \\
\hline $\mathrm{F} 2+\mathrm{F} 9=64.6302$ & Fx & 64.630 & 0.042 & $<50$ \\
\hline \multicolumn{5}{|l|}{ ID19 (Q4.2) } \\
\hline \multicolumn{5}{|l|}{$\mathrm{F} 6=0.5246=v_{\text {rot }}$} \\
\hline $2 \mathrm{~F} 6=1.0492$ & F35 & 1.0554 & 0.238 & 128 \\
\hline $3 \mathrm{~F} 6=1.5738$ & F75(Q5.1) & 1.5723 & 0.134 & 83 \\
\hline $\mathrm{F} 1-\mathrm{F} 6=14.0765$ & F52 & 14.0736 & 0.181 & 98 \\
\hline $\mathrm{F} 3-\mathrm{F} 6=26.2207$ & F24 & 26.2267 & 0.301 & 179 \\
\hline \multicolumn{5}{|l|}{ ID20 (Q3.2) } \\
\hline $2 \mathrm{~F} 1=10.2570$ & F54 & 10.2585 & 0.166 & 139 \\
\hline $\mathrm{F} 1+\mathrm{F} 2=13.6341$ & F16 & 13.6381 & 0.526 & 417 \\
\hline $\mathrm{F} 1+\mathrm{F} 3=11.2409$ & F54 & 11.2414 & 0.170 & 137 \\
\hline $\mathrm{F} 3-\mathrm{F} 1=0.9839$ & F35 & 0.9888 & 0.295 & 281 \\
\hline $\mathrm{F} 2+\mathrm{F} 3=14.6180$ & F48 & 14.6256 & 0.184 & 147 \\
\hline $\mathrm{F} 2-\mathrm{F} 1=3.3771$ & Fx & 3.374 & 0.070 & $<50$ \\
\hline \multicolumn{5}{|l|}{ ID24 (Q3.2) } \\
\hline $2 \mathrm{~F} 1=22.2024$ & $\mathrm{Fx}(\mathrm{LC})$ & 22.2022 & 0.064 & - \\
\hline
\end{tabular}

(F86 $=0.4577 \mathrm{~d}^{-1}$, Amp $=0.121 \mathrm{mmag}$, is $\left.\sim \mathrm{F} 18 / 2\right)$, but with unsuccessful results.

ID24 shows one low-frequency peak, F12 $=1.5832 \mathrm{~d}^{-1}$, among those of highest amplitude. This peak has been investigated for rotation using the 4-yr LC time series, but with unsuccessful results. Only the first harmonic of F1, 2F1, is detected and listed in Table 5. Moreover, a number of independent peaks with very low amplitudes are present in the low-frequency region.

The periodogram of ID33 is dominated by two main peaks in the 16-19 $\mathrm{d}^{-1}$ region. There are also three low-frequency peaks, F12 $=2.7085 \mathrm{~d}^{-1}, \mathrm{~F} 19=2.5964 \mathrm{~d}^{-1}$, and F20 $=4.6035 \mathrm{~d}^{-1}$, which are among those of highest amplitude (see Table B2). F12 and F19 seem to be independent pulsation modes. The first harmonic of F20 seems to be visible and was investigated for rotation. However, the full 4-yr LC data suggest that the first harmonic frequency does not match the observed peak F20. Moreover, this peak probably cannot be attributed to rotation because of its high frequency. Balona (2013) reported the presence of $v=1.432 \mathrm{~d}^{-1}$ and its first harmonic with amplitudes 0.103 and $0.047 \mathrm{mmag}$, which were attributed to rota- 
Table 6. $\delta$ Sct stars of Group III. ID: identification number; Lu09: identification number by Lu09; Qi.j: quarters; $N_{\mathrm{m}}$ : number of months observed; $N_{\mathrm{p}}$ : number of peaks detected with significance $\operatorname{sig}>50$. (a) $N>100$ except in Q8.1 (61 peaks) and Q8.3 (81 peaks); (b) $N>$ 100 except in Q8.1 (67 peaks) and Q8.3 (79 peaks).

\begin{tabular}{ccccc}
\hline ID & Lu09 & Qi.j & $N_{\mathrm{m}}$ & $N_{\mathrm{p}}$ \\
\hline 02 & V6 & $2.1,3.2,5,6,11,13$ & 14 & $>100$ \\
05 & V1 & $2.3,5,8.1,8.3,9-11$ & 15 & $>100$ (a) \\
28 & V5 & $1.3,5,7-11$ & 19 & $>100(\mathrm{~b})$ \\
36 & V2 & $2.3,5-10$ & 19 & $\sim 100$ \\
\hline
\end{tabular}

tion. These peaks are confirmed in our analysis on the full LC time series, giving $v_{1}=1.4321 \mathrm{~d}^{-1}, v_{2}=2.8705 \mathrm{~d}^{-1}$ with amplitudes 0.090 and 0.045 mmag. However, the temporal evolution of these two peaks using LC data is somewhat different from each other.

The analysis of the two remaining stars in Group II, ID12 and ID20, does not show any low-frequency peak among the peaks of highest amplitude that could be attributed to rotation. ID20 was also unsuccessfully investigated for rotation by Balona (2013). Fig. 6 shows their very different periodograms. The main oscillations of ID12 all have frequencies larger than $14 \mathrm{~d}^{-1}$. On the other hand, peaks below $10 \mathrm{~d}^{-1}$ dominate the pulsations of ID20. A few frequency combination frequencies with $\mathrm{F} 1$ as a parent mode can be identified, as shown in Table 5. A number of very low amplitude peaks in the g-mode regime are also confirmed for both stars using the $\mathrm{LC}$ time series.

\section{GROUP III: $\delta$ SCT STARS WITH MORE THAN 7 MONTHS OF SC OBSERVATIONS}

Group III consists of four $\delta$ Sct stars observed in SC mode for 14 19 months (see Table 6). They were discovered as $\delta$ Sct variables by van Cauteren et al. (2005) and confirmed by Luo et al. (2009). In nearly all quarters the number of significant peaks is larger than 100. The only exceptions are ID05 and ID28 for quarters Q8.1 and Q8.3, where only 18 and $20 \mathrm{~d}$ of data are available compared with about $30 \mathrm{~d}$ in the other quarters. Table B3 lists the results for the 20 peaks of highest amplitude and the first available SC quarter Qi.j. Frequency identifications of interest are reported in Table 7, except for ID36 that will be analysed in more detail at the end of the section.

ID02 is a $\delta$ Sct star with the largest luminosity variations in the entire sample. The frequencies in this star are relatively low, being mostly in the $4-10 \mathrm{~d}^{-1}$ range. The periodogram is dominated by an isolated peak at $\mathrm{F} 1=9.9558 \mathrm{~d}^{-1}$ with an amplitude of 29 mmag. Some harmonics together with some frequency combinations involving F1 and F2-F4 are detected and listed in Table 7. A number of independent peaks of low amplitude are found below $2 \mathrm{~d}^{-1}$. No evidence of rotational modulation was found.

For ID05, only two combination frequencies with very low amplitude, F1 + F2 and F2 - F1, were detected. There is one low-frequency peak, F13 $=1.4911 \mathrm{~d}^{-1}$, among the set of 20 peaks of highest amplitude. This is close to F3 $-\mathrm{F} 10=1.4883 \mathrm{~d}^{-1}$, but there seems to be no direct connection between these two peaks. This suggests F3 as an independent pulsation peak in the $\gamma$ Dor regime. Balona (2013) reported peaks $v=0.279 \mathrm{~d}^{-1}$ and its harmonic, with amplitudes 0.073 and $0.043 \mathrm{mmag}$, respectively, which were attributed to rotation. These are confirmed when we use the full LC time series.
Table 7. Identifications of interest for $\delta$ Sct stars of Group III. For each star, the corresponding first month Qi.j of SC observations is indicated. Parentheses note when the peaks come from a different quarter. Fx: peaks with significance sig $<50$ or from the full LC time series.

\begin{tabular}{|c|c|c|c|c|}
\hline $\begin{array}{l}\text { Iden } \\
\left(d^{-1}\right)\end{array}$ & $\mathrm{Fi}$ & $\begin{array}{l}\text { Freq } \\
\left(d^{-1}\right)\end{array}$ & $\begin{array}{c}\text { Amp } \\
(\mathrm{mmag})\end{array}$ & Sig \\
\hline \multicolumn{5}{|l|}{ ID02 (Q2.1) } \\
\hline $2 \mathrm{~F} 1=19.9116$ & F18 & 19.9119 & 1.124 & 519 \\
\hline $3 \mathrm{~F} 1=29.8674$ & F93 & 29.8679 & 0.260 & 122 \\
\hline $4 \mathrm{~F} 1=39.8232$ & Fx & 39.821 & 0.110 & $<50$ \\
\hline$F 1+F 2=16.0104$ & F28 & 16.0097 & 0.646 & 260 \\
\hline $\mathrm{F} 1-\mathrm{F} 2=3.9012$ & $\mathrm{Fx}(\mathrm{LC})$ & 3.9022 & 0.079 & - \\
\hline $\mathrm{F} 1+\mathrm{F} 3=17.4011$ & $\mathrm{~F} 23$ & 17.4013 & 0.795 & 333 \\
\hline $\mathrm{F} 1-\mathrm{F} 3=2.5105$ & F66 & 2.5127 & 0.322 & 132 \\
\hline $\mathrm{F} 1+\mathrm{F} 4=16.8240$ & F38 & 16.8239 & 0.465 & 172 \\
\hline $\mathrm{F} 1-\mathrm{F} 4=3.0876$ & $\mathrm{Fx}(\mathrm{LC})$ & 3.0867 & 0.080 & - \\
\hline \multicolumn{5}{|l|}{ ID05 (Q2.3) } \\
\hline$v_{\mathrm{rot}}=0.2792(\mathrm{LC})$ & F39 & 0.2701 & 0.195 & 108 \\
\hline $2 v_{\mathrm{rot}}=0.5584$ & $\operatorname{Fx}(\mathrm{LC})$ & 0.5570 & 0.046 & - \\
\hline $3 v_{\mathrm{rot}}=0.8376$ & $\operatorname{Fx}(\mathrm{LC})$ & 0.8374 & 0.010 & - \\
\hline$F 1+F 2=44.4861$ & $\mathrm{Fx}(\mathrm{Q} 5.1)$ & 44.480 & 0.021 & $<50$ \\
\hline $\mathrm{F} 2-\mathrm{F} 1=3.3495$ & F84(Q5.1) & 3.3388 & 0.094 & 69 \\
\hline \multicolumn{5}{|l|}{ ID28 (Q1.3) } \\
\hline \multirow[t]{2}{*}{$v_{\mathrm{rot}}=0.5511(\mathrm{LC})$} & F33 & 0.5509 & 0.140 & 202 \\
\hline & $\mathrm{Fx}(\mathrm{LC})$ & 0.5511 & 0.090 & - \\
\hline \multirow[t]{2}{*}{$2 v_{\mathrm{rot}}=1.1022$} & Fx & 1.106 & 0.044 & $<50$ \\
\hline & $\operatorname{Fx}(\mathrm{LC})$ & 1.1021 & 0.029 & - \\
\hline $2 \mathrm{~F} 1=30.3524$ & Fx & 30.358 & 0.055 & $<50$ \\
\hline $\mathrm{F} 1-\mathrm{F} 2=2.6359$ & Fx & 2.626 & 0.080 & $<50$ \\
\hline
\end{tabular}

The $\delta$ Sct pulsations in ID28 are mainly in the range $11-20 \mathrm{~d}^{-1}$. Only two frequency combinations with very low amplitude, $2 \mathrm{~F} 1$ and $\mathrm{F} 1-\mathrm{F} 2$, are found among the main pulsation peaks. There are a number of independent peaks in the low-frequency domain. There is a sharp peak at $\mathrm{F} 33=0.5511 \mathrm{~d}^{-1}$, flanked by a broad peak centred at slightly lower frequency at about $0.51 \mathrm{~d}^{-1}$, as shown in Fig. 9. This is an example of the peculiar periodogram feature of the ROTD variables described by Balona (2013, 2014c, 2017) among A- and B-type stars. This strange feature is quite common among A stars and is also known as 'hump and spike' (Saio et al. 2018). Balona (2014a) suggested that the broad peak is due to differential rotation, while the sharp peak could be the result of a reflection effect from a non-transiting planet in a synchronous orbit. However, Saio et al. (2018) proposed a more likely explanation: the sharp peak is the rotational frequency, while the broad peak is due to Rossby waves.

Fig. 9 also shows a similar peculiar feature in ID36, but the nature of the sharp F6 peak seems to be different from that of F33 in ID28. The sharp peak in ID28 has constant amplitude and frequency, within the uncertainties, over the whole $4 \mathrm{yr}$ of LC observations. On the other hand, the sharp peak F6 in ID36 is much broader with variable amplitude, resembling what might be expected from a starspot. Also, the amplitude of $\mathrm{F} 6(\approx 0.8 \mathrm{mmag})$ is rather large for a reflection effect of a planet. This would imply a minimum radius of $\approx 4.5 R_{\text {Jup }}$, using the relations in Balona (2014c). The typical amplitude of the sharp peak of the known ROTD variables is of only $\approx 0.02$ mmag (Balona 2017).

The amplitude stability of the principal pulsation frequencies for ID02, ID05, and ID28 was investigated using all available SC observations by fitting the 20 frequencies of largest amplitude to each quarter. The resulting amplitudes are presented in Fig. 10 for the 10 frequencies of highest amplitude. A number of peaks show 

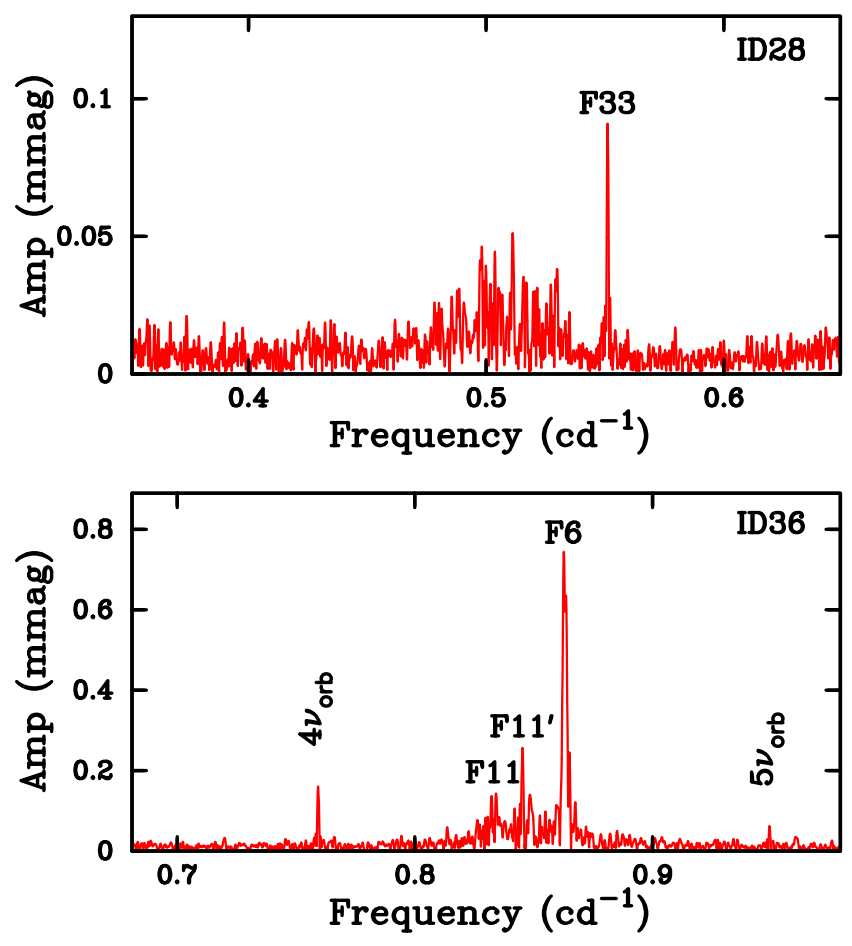

Figure 9. ID28 and ID36 periodograms, for the 4-yr LC time series, in the region of the peculiar ROTD features, showing the peaks F33 $=0.5511 \mathrm{~d}^{-1}$ (ID28) and F6 $=0.8626 \mathrm{~d}^{-1}$ together with F11 $=0.8341 \mathrm{~d}^{-1}$ and F11 $=0.8452 \mathrm{~d}^{-1}$ (ID36). However, the nature of the two peaks F33 and F6 is different (see text for details). The peaks corresponding to $4 v_{\text {orb }}$ and $5 v_{\text {orb }}$ for ID36 are also shown.

significant amplitude variability in all three stars. This amplitude variability in $\delta$ Sct stars has already been noted by Bowman et al. (2016), who also included ID05 and ID28 in their analysis.

It is interesting to note for ID28 the continuous decrease in amplitude of F1 during more than 20 months, with a rate of amplitude decrease of $\sim 0.12 \mathrm{mmag}^{\text {month }}{ }^{-1}$ (which could be produced by some non-linear effect) or the apparently sinusoidal variability of F10 with a periodicity of $\sim 500 \mathrm{~d}$ (which might be produced by an unresolved pulsation mode contained by an extremely close frequency pair, separated $\sim 0.002 \mathrm{~cd}^{-1}$ from each other). Nevertheless, a much more detailed study is needed, including the analysis of the full LC time series together with possible associated phase variations, to determine the true nature of each of these variations. However, this falls out of the scope of the present work.

\subsection{ID36}

In this section, we present a summary of our findings on the remarkable star ID36. A more detailed discussion will be presented elsewhere.

The light curve and periodogram of ID36 are shown in Fig. 6. Table B3 lists the frequencies and amplitudes of the 20 peaks of maximum amplitude for SC data in quarter Q2.3. The $\delta$ Sct peaks are mainly confined to the small range $18-22 \mathrm{~d}^{-1}$. A number of significant peaks are also present in the low-frequency domain. These may be a result of binarity $\left(\mathrm{F} 2=0.1900 \mathrm{~d}^{-1}, \mathrm{~F} 8=2 \mathrm{~F} 2\right)$ or stellar rotation $\left(\mathrm{F} 6=0.8616 \mathrm{~d}^{-1}, \mathrm{~F} 11=0.8329 \mathrm{~d}^{-1}\right)$. The last column in Table B3 gives the possible identification for many of the main peaks. Only four peaks, F1, F3, F4, and F7, of the 12 peaks of

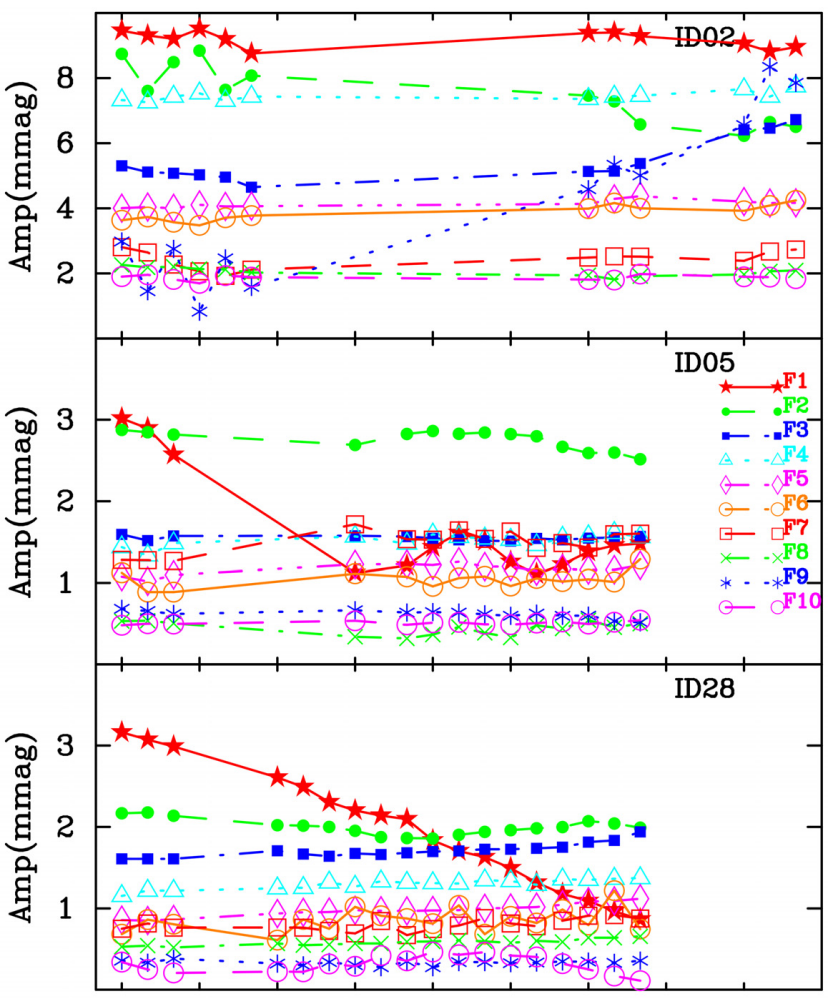

Q5.1 Q6.1 $\quad$ Q7.1 $\quad$ Q8.1 $\quad$ Q9.1 Q10.1 Q11.1 Q12.1 Q13.1

Quarters (Qi.j)

Figure 10. ID02 (top), ID05 (middle), and ID28 (bottom): temporal evolution of the amplitudes for the 10 main pulsation modes throughout the available SC quarters, starting from Q5.1. For clarity, F1 amplitudes for ID02 have been offset -18 mmag in the $y$-axis. A number of peaks show variable amplitude for each star. Note that the typical formal error bars of the amplitude points are much smaller than the symbols in the figure (see Table B3).

highest amplitude, seem to be independent modes. The rest appear to be related to the orbital frequency of the system or stellar rotation.

In Table 8, we present a number of possible frequency identifications including a few frequency combination of very low amplitude involving F1. Moreover, the majority of the combinations involve F2 or its harmonics or an interaction with the four main pulsation peaks. These could be interpreted as the first- and second-order sidelobes produced by the orbital motion on the pulsating modes. The binary character of F2 is also supported by the sharp appearance of the peak in the periodogram, and its amplitude stability as derived using all available SC data, as shown in Fig. 11. On the other hand, some additional independent low-amplitude modes in the low-frequency region were also detected, typical of pulsations in the g-mode regime.

\subsubsection{Amplitude variability in ID36}

The variation of amplitude with time for the principal frequencies in ID36 was investigated using the whole set of 19 months of available SC data. This star was not analysed by Bowman et al. (2016). Results are shown in Fig. 11 for the four main pulsation peaks, F1, F3, F4, F7, and also for F2 and F6. The amplitudes of all four main pulsation peaks vary with periodicities of about $9,6,3$, and 6 months, respectively. 
Table 8. Identifications of interest for ID36 and quarter Q2.3. Fx: peaks with sig $<50$ significance. In parentheses if it is a different quarter.

\begin{tabular}{|c|c|c|c|c|}
\hline $\begin{array}{l}\text { Iden } \\
\left(\mathrm{d}^{-1}\right)\end{array}$ & $\mathrm{Fi}$ & $\begin{array}{l}\text { Freq } \\
\left(d^{-1}\right)\end{array}$ & $\begin{array}{c}\text { Amp } \\
(\mathrm{mmag})\end{array}$ & Sig \\
\hline $2 \mathrm{~F} 1=43.2262$ & F60 & 43.2288 & 0.051 & 101 \\
\hline $\mathrm{F} 1+\mathrm{F} 3=40.6347$ & F72 & 40.6329 & 0.040 & 69 \\
\hline $\mathrm{F} 1-\mathrm{F} 3=2.5915$ & F65 & 2.5887 & 0.046 & 87 \\
\hline \multirow[t]{2}{*}{$\mathrm{F} 1+\mathrm{F} 4=42.2271$} & Fx & 42.226 & 0.034 & $<50$ \\
\hline & F83(Q5.2) & 42.2230 & 0.041 & 77 \\
\hline \multicolumn{5}{|l|}{$\mathrm{F} 2=0.1900=v_{\mathrm{orb}}$} \\
\hline $2 \mathrm{~F} 2=0.3800$ & F8 & 0.3789 & 0.542 & 1218 \\
\hline $3 \mathrm{~F} 2=0.5700$ & $\mathrm{~F} 25$ & 0.5732 & 0.152 & 403 \\
\hline $4 \mathrm{~F} 2=0.7600$ & F23 & 0.7652 & 0.187 & 511 \\
\hline $5 \mathrm{~F} 2=0.9500$ & F45 & 0.9485 & 0.070 & 139 \\
\hline $6 \mathrm{~F} 2=1.1400$ & F75 & 1.1406 & 0.037 & 63 \\
\hline $\mathrm{F} 1+\mathrm{F} 2=21.8031$ & F17 & 21.8056 & 0.252 & 583 \\
\hline $\mathrm{F} 1-\mathrm{F} 2=21.4231$ & F12 & 21.4276 & 0.333 & 719 \\
\hline $\mathrm{F} 1+2 \mathrm{~F} 2=21.9931$ & F56 & 21.9914 & 0.056 & 112 \\
\hline \multirow[t]{2}{*}{$\mathrm{F} 1-2 \mathrm{~F} 2=21.2331$} & F44(Q5.1) & 21.2309 & 0.079 & 186 \\
\hline & F39(Q5.2) & 21.2333 & 0.116 & 253 \\
\hline $\mathrm{F} 3+\mathrm{F} 2=19.2116$ & F19 & 19.2104 & 0.226 & 512 \\
\hline $\mathrm{F} 3-\mathrm{F} 2=18.8316$ & F5 & 18.8302 & 0.909 & 1793 \\
\hline \multirow[t]{2}{*}{$\mathrm{F} 3+2 \mathrm{~F} 2=19.4016$} & F35 & 19.4013 & 0.083 & 182 \\
\hline & F65(Q5.1) & 19.4070 & 0.063 & 127 \\
\hline $\mathrm{F} 4+\mathrm{F} 2=20.8040$ & F53 & 20.7971 & 0.061 & 122 \\
\hline \multirow[t]{2}{*}{$\mathrm{F} 4-\mathrm{F} 2=20.4240$} & F57 & 20.4381 & 0.055 & 112 \\
\hline & F37(Q5.1) & 20.4296 & 0.113 & 296 \\
\hline $\mathrm{F} 4+2 \mathrm{~F} 2=20.9940$ & F68 & 20.9981 & 0.045 & 85 \\
\hline $\mathrm{F} 7+\mathrm{F} 2=20.3300$ & F9 & 20.3265 & 0.400 & 837 \\
\hline $\mathrm{F} 7-\mathrm{F} 2=19.9500$ & F10 & 19.9529 & 0.394 & 787 \\
\hline \multirow[t]{2}{*}{$\mathrm{F} 7+2 \mathrm{~F} 2=20.5200$} & F84 & 20.5320 & 0.033 & 53 \\
\hline & F45(Q5.1) & 20.5209 & 0.085 & 189 \\
\hline \multirow{2}{*}{$\mathrm{F} 7-2 \mathrm{~F} 2=19.7600$} & F43 & 19.7576 & 0.078 & 161 \\
\hline & F47(Q5.1) & 19.7550 & 0.085 & 193 \\
\hline$v_{\text {rot }}=0.8626(\mathrm{LC})$ & F6 & 0.8616 & 0.579 & 1683 \\
\hline $2 v_{\mathrm{rot}}=1.7252$ & $\operatorname{Fx}(\mathrm{LC})$ & 1.7259 & 0.089 & - \\
\hline $3 v_{\text {rot }}=2.5878$ & $\operatorname{Fx}(\mathrm{LC})$ & 2.5877 & 0.011 & - \\
\hline
\end{tabular}

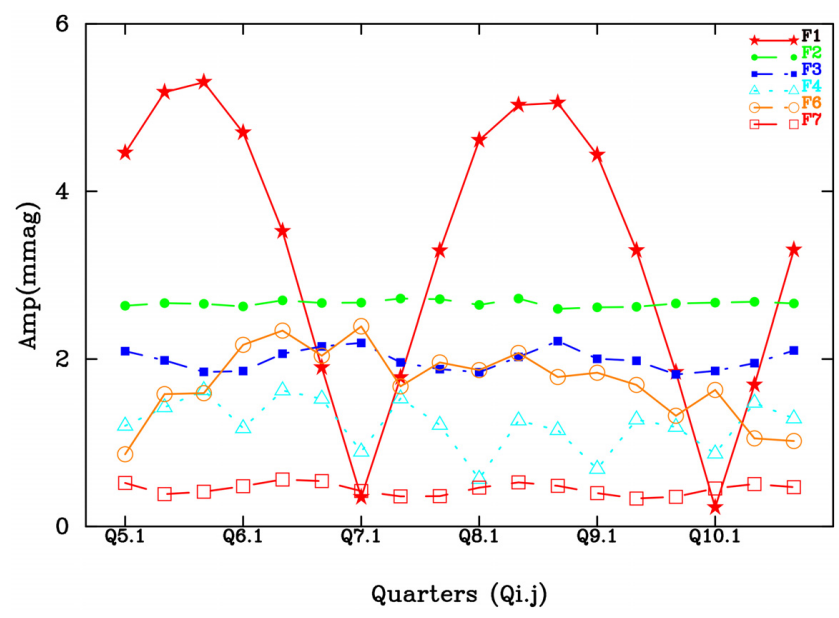

Figure 11. ID36: temporal evolution of the amplitudes of the main periodicities throughout the SC mode quarters.

These periodic amplitude variations might be the result of unresolved peaks that might be detected with the full 4-yr LC data. In fact, two very closely spaced peaks around F1 are detected, $F 1^{\prime}=21.6113 \mathrm{~d}^{-1}$ and $\mathrm{F} 1^{\prime \prime}=21.6150 \mathrm{~d}^{-1}$ with very similar amplitudes consistent with the amplitude modulation shown in
Fig. 11. However, these two peaks could be two independent pulsation modes with constant amplitudes or the result of only one peak that is split into two due to real amplitude variations. A more detailed investigation is needed and will be performed in a forthcoming work.

\subsubsection{Binarity in ID36}

Table 8 shows that frequency combinations involving the orbital frequency, F2, and its harmonics and the principal pulsation modes are clearly present. The best way to investigate the multiplets introduced by the orbital frequency is to construct an echelle diagram for ID36. This is shown in Fig. 12, as a result of the frequency content of the periodogram when the 4-yr LC time series is analysed. This diagram shows the fundamental orbital frequency and up to six harmonics (vertical line of dots at zero) in the low-frequency region. The other points, unrelated to the orbital frequency, are related to rotation or g-mode pulsations.

In the high-frequency region, we can see many equally spaced points along vertical lines. These are frequencies spaced by multiples of $v_{\text {orb }}$. They cannot be simply harmonics of the orbital frequency (because they have the form $v=n v_{\text {orb }}+v_{0}(n= \pm 1$, $2,3, \ldots)$, where $v_{0}$ is the pulsation frequency), nor combination frequencies (because all of them are exactly separated by $v_{\text {orb}}$ ). Also, they cannot be interpreted as sidelobes produced by the timedelay effect in a binary system because the orbital period is too short and the expected amplitudes of the sidelobes are too small to be detected (see Shibahashi \& Kurtz 2012; Balona 2014d).

The only plausible interpretation is tidal effects in a binary system where the pulsating component is tidally distorted, as discussed in Balona (2018b). In this case, we expect to see the so-called 'ellipsoidal' variations because the star is no longer a sphere, so the brightness varies with twice the orbital frequency (half the orbital period). If reflection effects are important, the symmetry is broken and the peaks will happen at $n v_{\text {orb }}$, as seems to be the case in ID36.

In addition to rotation, tidal forces in a binary introduce another axis of symmetry joining the two centres of mass. The observed equally spaced multiplets are most likely a result of the variable viewing angle along the orbit. We can recover the light curve due to the tidal effect by fitting and removing the frequencies not associated with $v_{\text {orb }}$ and its harmonics. The resulting light curve is phased with the orbital period of $P_{\text {orb }}=5.267954 \mathrm{~d}$ is shown in Fig. 13 .

\subsubsection{Activity in ID36}

Both F6 and F11 appear to be related to rotation. The periodogram is shown in Fig. 9 and the amplitude variation in Fig. 14. Because of changes in amplitude and frequency, we fix the values of these two frequencies to the mean values obtained from the full 19 months of $\mathrm{SC}$ data as given in Table B3. From the complete 4-yr LC data, the frequencies are $\mathrm{F} 6=0.8626 \mathrm{~d}^{-1}$ and $\mathrm{F} 11=0.8341 \mathrm{~d}^{-1}$. The second and third harmonics of $\mathrm{F} 6$ are also visible and listed in Table 8. A third peak at $\mathrm{F} 11^{\prime}=0.8452 \mathrm{~d}^{-1}$, which cannot be resolved using the monthly SC data, is also evident in the LC periodogram.

F6 amplitude variation in Fig. 14 suggests that it may be a result of a well-defined star-spot close to the equator, which is highly variable in size. The origin of F11 and F11' may be a single common zone of activity on the stellar surface. Both activity zones probably belong to the same component of the binary system. The activity region connected to F11 and F11' is located at a higher latitude than F6, which is consistent with a difference of about 3 per cent in period due 

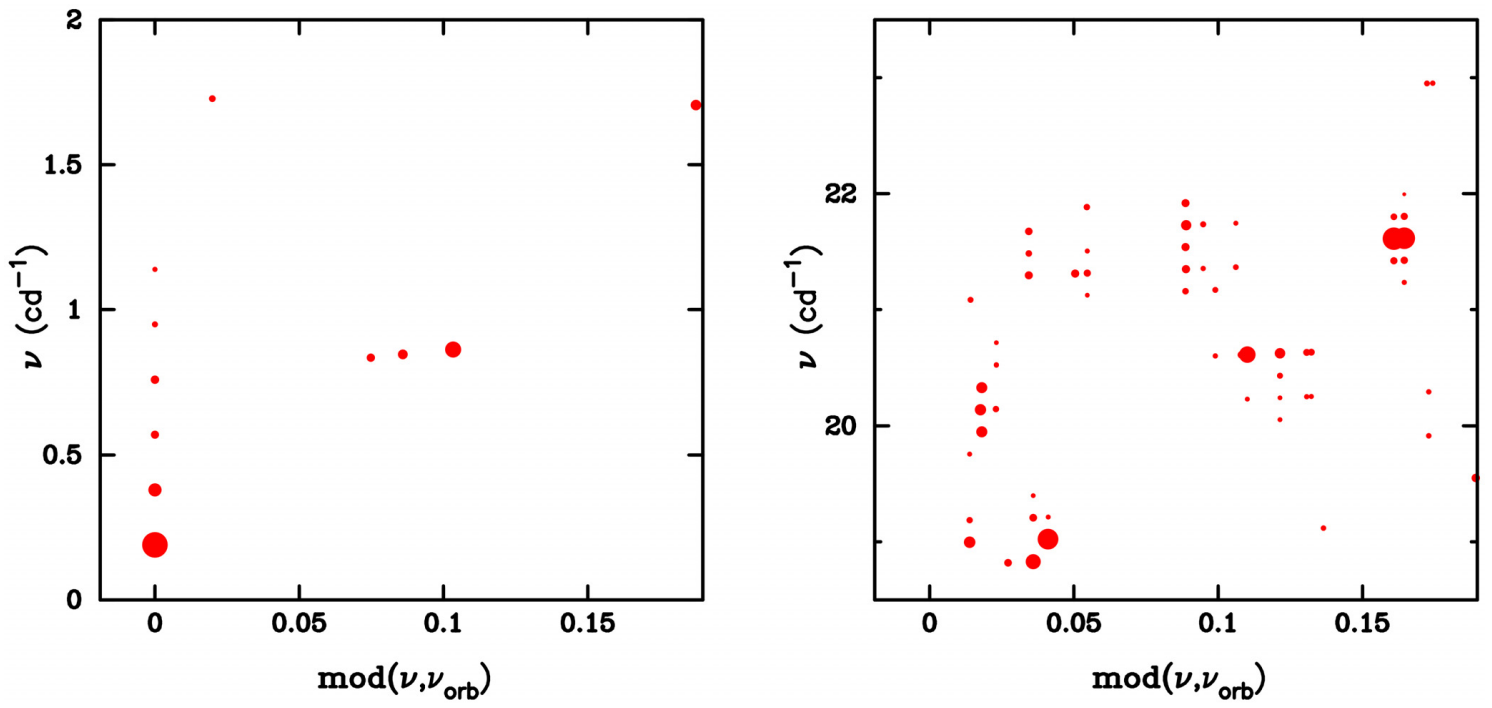

Figure 12. ID36: echelle diagram in the low-frequency (left) and high-frequency (right) regions using $v_{\text {orb }}=0.189827 \mathrm{~d}^{-1}$. The size of the symbols is proportional to the amplitude.

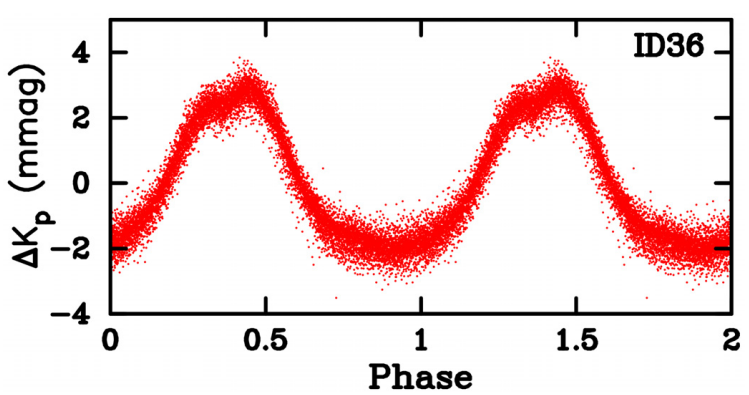

Figure 13. ID36: the light curve with all variations removed except for the orbital frequency and its harmonics phased with $P_{\text {orb }}=5.267954 \mathrm{~d}$.

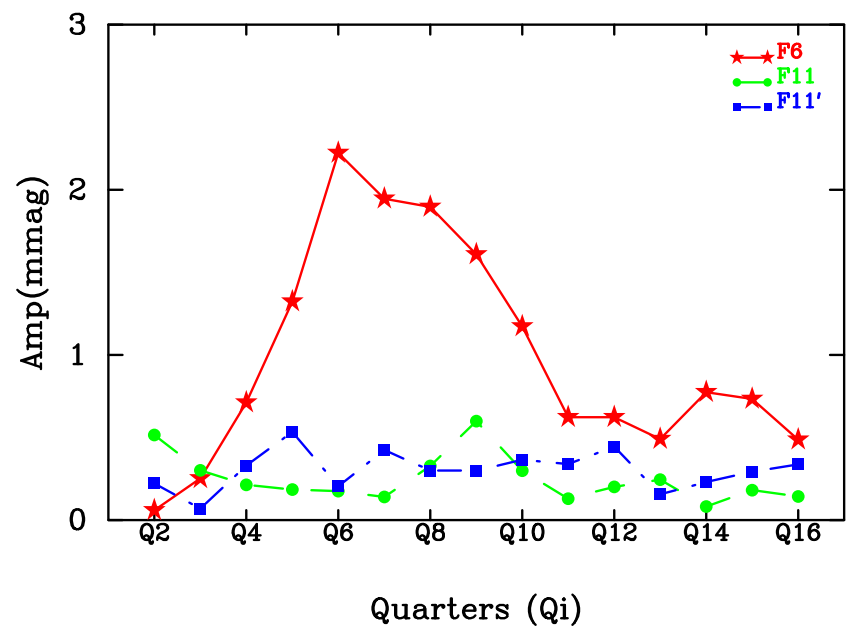

Figure 14. ID36: temporal evolution, over the 4-yr LC time series, of the amplitudes of F6 $=0.8626 \mathrm{~d}^{-1}, \mathrm{~F} 11=0.8341 \mathrm{~d}^{-1}$ and $\mathrm{F} 11^{\prime}=0.8452 \mathrm{~d}^{-1}$.

to differential rotation. A number of possible interactions between $v_{\text {rot }}$ and the main $\delta$ Sct pulsation modes are also visible, as shown in Table 9, suggesting that the active star in ID36 is the pulsating component.
Table 9. ID36: identified interactions between $v_{\text {rot }}\left(=0.8626 \mathrm{~d}^{-1}, \mathrm{LC}\right)$ and the main $\delta$ Sct-type pulsation modes, $\mathrm{F}^{\prime}=21.6113 \mathrm{~d}^{-1}$ and $\mathrm{F} 1^{\prime \prime}$ $=21.6150 \mathrm{~d}^{-1}$, using the $\mathrm{LC}$ time series.

\begin{tabular}{lcc}
\hline $\begin{array}{l}\text { Iden } \\
\left(\mathrm{d}^{-1}\right)\end{array}$ & $\begin{array}{c}\text { Freq } \\
\left(\mathrm{d}^{-1}\right)\end{array}$ & $\begin{array}{c}\text { Amp } \\
(\mathrm{mmag})\end{array}$ \\
\hline $\mathrm{F} 1^{\prime}+v_{\text {rot }}=22.4739$ & 22.4758 & 0.009 \\
$\mathrm{~F} 1^{\prime \prime}+v_{\text {rot }}=22.4776$ & 22.4797 & 0.010 \\
$\mathrm{~F} 1^{\prime}-v_{\text {rot }}=20.7487$ & 20.7460 & 0.028 \\
$\mathrm{~F}^{\prime \prime}-v_{\text {rot }}=20.7524$ & 20.7507 & 0.029 \\
\hline
\end{tabular}

Interestingly, flares are also present in this system. They are detected by visual inspection of the residuals SC light curves after the first 100 significant peaks are extracted from the periodograms (Figs 15 and 16). Each flare was cross-checked with the light curves of other stars observed during the same quarters as ID36, to rule out any instrumental effect. The relative large amplitude short-period light variation present in the pulsating component of ID36 means that flares can only be detected when most of the periodicities have been removed from the original light curve. It is sometimes difficult to estimate exactly when one flare ends due to the pulsational variability in ID36. This means that many other microflares may be present in the light curves, but are undetectable. The flares are probably taking place in the pulsating component of ID36, as this is the component where stellar activity appears to be present.

All the presumed flaring events listed in Table 10 have the typical characteristics of flares, i.e. a very rapid rise in brightness followed by a much slower decay. The duration commonly ranges between 20 and $50 \mathrm{~min}$, with very rapid increases that take only about 4-6 min. The full width at half-maximum (FWHM) intervals last about 3050 per cent of the total duration of each flare. A total of 10 flares were detected during the 18 months of continuous SC observations of ID36. This means one flare every $54 \mathrm{~d}$. However, they are not regularly distributed in time. Sometimes the time interval between flares is just a few days (e.g. Q7.2-Q7.3 or Q5.1), but there are also long periods of inactivity of more than $200 \mathrm{~d}$ (e.g. between Q5.1 and Q7.2). In summary, it seems there are epochs with violent episodes 


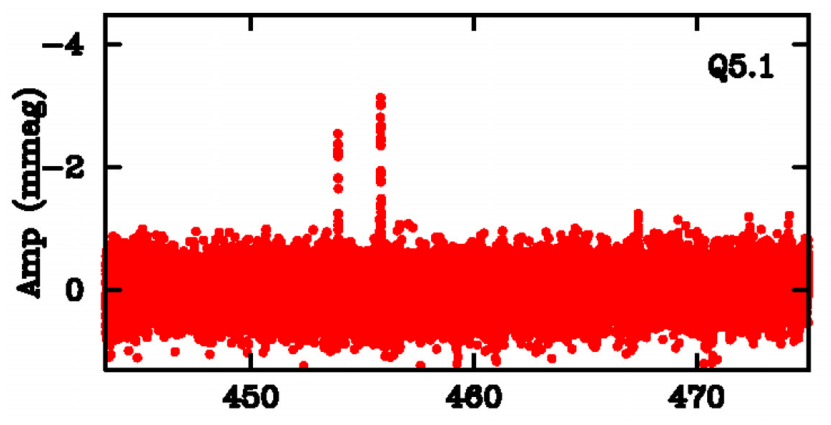

BJD-2454838.0 +

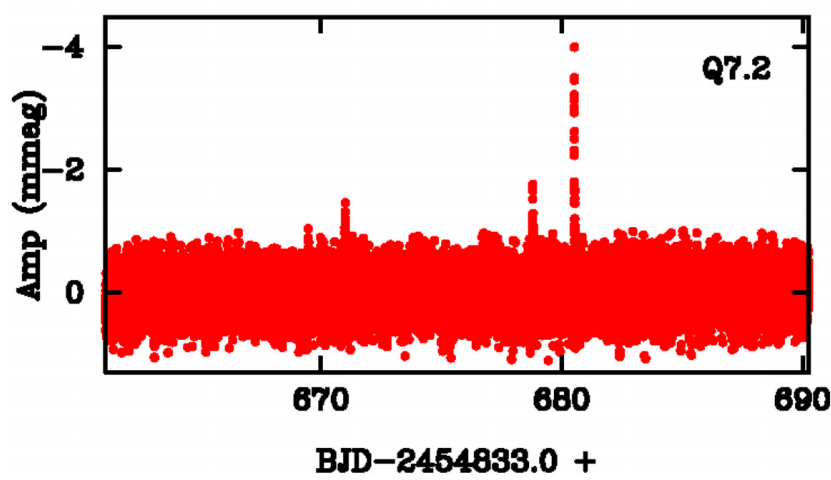

Figure 15. ID36: examples of flares in the residuals of the SC light curves after removing 100 peaks.
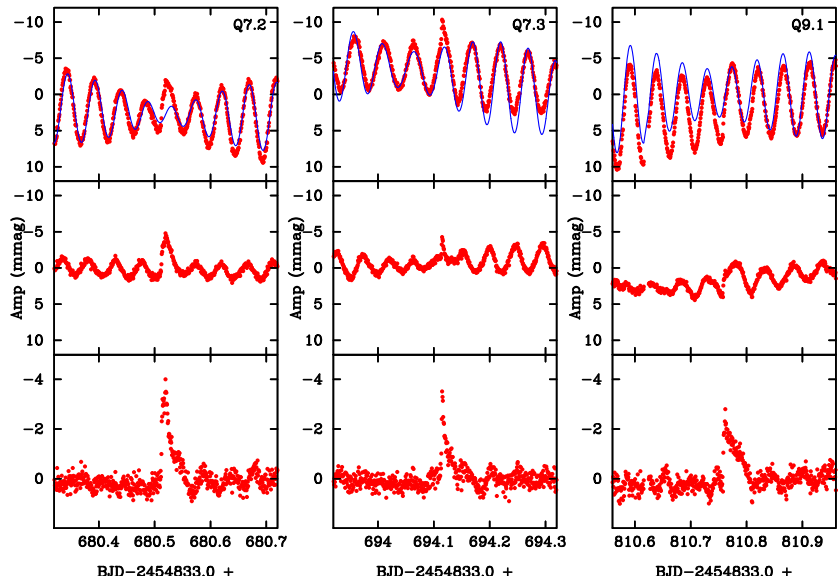

Figure 16. ID36: examples of SC light curves with flares. Top panels: original light curves and fits with the first 20 peaks; middle panels: residuals after removing 20 peaks; bottom panels: residuals after removing 100 peaks.

followed by long periods of inactivity, as it usually happens in cool M dwarfs.

Following Walkowicz et al. (2011) and Balona (2015), we can estimate the energy emitted during the flares in ID36 (see Table 10). A reduction factor of 0.45 was applied to take into account the fraction of quiescent luminosity emitted by the star through the Kepler passband. This results in a typical energy of about $10^{35} \mathrm{erg}$, i.e. $10^{6}$ times the energy emitted in a typical solar flare or about $10^{3}-$ $10^{4}$ times the energy emitted during the most intense solar flares (Benz \& Güdel 2010). Therefore, they are superflares as typically encountered in flaring A stars detected by the Kepler mission.
Table 10. Flares detected in the SC light curves of ID36. The quarters Qi.j, times of maximum relatives to BJD $=2454833.0+$, amplitudes, and estimated durations are listed together with the integrated flux, $E W(\mathrm{~h})$, and flare energy, $E$ (erg).

\begin{tabular}{lccccc}
\hline $\mathrm{Q}$ & $\begin{array}{c}T_{\max } \\
(\mathrm{d})\end{array}$ & $\begin{array}{c}\text { Amp } \\
(\mathrm{mmag})\end{array}$ & $\begin{array}{c}\Delta T \\
(\mathrm{~min})\end{array}$ & $\log E W$ & $\log E$ \\
\hline 5.1 & 453.902 & 2.5 & 28 & -3.284 & 34.88 \\
5.1 & 455.818 & 3.0 & 41 & -2.996 & 35.17 \\
7.2 & 670.999 & 1.5 & 46 & -3.301 & 34.87 \\
7.2 & 678.787 & 1.8 & 42 & -3.252 & 34.92 \\
7.2 & 680.520 & 4.0 & 57 & -2.757 & 35.41 \\
7.3 & 694.116 & 3.5 & 34 & -3.114 & 35.06 \\
8.2 & 766.853 & 1.6 & 31 & -3.347 & 34.82 \\
9.1 & 810.761 & 2.5 & 52 & -2.936 & 35.23 \\
10.1 & 908.026 & 1.6 & 16 & -3.699 & 34.47 \\
10.2 & 967.935 & 1.8 & 33 & -3.319 & 34.85 \\
\hline
\end{tabular}

\section{DISCUSSION}

We have analysed a selected sample of 36 Kepler SC stars in the field of NGC 6811, the majority of them being members of the cluster. The results reveal that all the targets are variable: 23 of them are pulsators, 21 main-sequence $\delta$ Sct- (16) or $\gamma$ Dor-type (5) pulsators together with two red giant stars with solar-like oscillations. All 13 remaining stars are found to be rotating variables. This means that activity in the stellar surface is commonly taking place, even for the hotter stars in the sample.

The periodograms of the two red giants in the sample (Fig. 3) show a sharp increase in power towards low frequencies suggesting surface activity. If the cause is rotation, this would suggest rotational periodicities in the range $\sim 40-50 \mathrm{~d}$. Nevertheless, in red giants, the convection cells are large and can dominate the light variations. Hence, the cause could probably be convection rather than rotation.

Five new rotating variables are detected in this work: ID01, ID15, ID16, ID23, and ID30. One of them, ID23, with a rotational period of $0.98 \mathrm{~d}$ and very small photometric amplitude, Amp $=0.02 \mathrm{mmag}$, appears as one of the hotter stars in the sample.

Three out of five $\gamma$ Dor stars in the sample are detected as new $\gamma$ Dor-type variables: ID26, ID34, and ID35. One of them, ID34, is one hot $\gamma$ Dor star similar to those discussed in Balona et al. (2016). In fact, ID34 is the hottest star in our sample. In all the $\gamma$ Dor stars, except in ID08, we detect rotational modulation. In some of them, surface activity dominates the luminosity variations. On the other hand, none of the five $\gamma$ Dor stars show any significant peak in the high-frequency domain $\left(v>5 \mathrm{~d}^{-1}\right)$, in good agreement with previous results (e.g. Balona 2014a, 2018c). Interestingly, two $\gamma$ Dor stars, ID08 and ID31, show similar sequences of peaks of almost equal separation that deserve closer investigation. They probably are sequences of dipole gravity g-modes as seen in KIC 5123889 (Balona 2018a).

Many of the $\delta$ Sct stars analysed here show very dense frequency distributions. The number of significant peaks is typically larger than 100 when the amplitude of the main peak is larger than about $2 \mathrm{mmag}$. There is a wide diversity in frequency patterns, even for stars that are members of NGC 6811, which presumably have similar ages and abundances, and with very similar location in the H-R diagram. Examples are (ID03, ID20), (ID05, ID21), (ID24, ID28), and (ID14, ID33). The large differences in frequency distributions in Kepler $\delta$ Sct stars with similar physical parameters have previously been noted by Balona et al. (2015). It seems that small differences in the fundamental parameters may lead to large 
differences in frequency distribution, suggesting that non-linearity and mode selection in the envelopes may play a very important role. Interestingly, there are also some couples of stars, (ID14, ID34) or (ID02, ID29), where one star is a $\delta$ Sct pulsator, but the other one is not pulsating (ID29) or pulsating only in the low-frequency domain (ID34).

All the $\delta$ Sct stars in our sample are $\delta$ Sct $/ \gamma$ Dor hybrids, confirming the already known result that over 98 per cent of $\delta$ Sct stars are 'hybrids' (Balona 2018c). For all stars, most peaks in the low-frequency region are independent modes. In a number of stars, some of the peaks in the low-frequency region are among those of highest amplitude. Hence, the definition of a $\gamma$ Dor star is simply a $\delta$ Sct star where no significant frequencies are present above $5 \mathrm{~d}^{-1}$. There appears to be no real difference between the two classes of stars. $\gamma$ Dor, $\delta$ Sct, and constant stars are to be found within the same small region at the cool end of the instability strip (Balona 2018c).

Many of the $\delta$ Sct stars discussed above seem to exhibit rotational modulation (Table 1), as shown by the presence of a low-frequency peak and its harmonic, both of which tend to be variable in amplitude. This is an indication on the existence of stellar activity, either through star-spots or any other corotating surface inhomogeneity. These rotational peaks are, in many cases, among the peaks with the largest amplitudes. Moreover, rotational modulation dominates the photometric variability of some $\gamma$ Dor stars in our sample.

We detect rotation in nine out of $16 \delta$ Sct stars, i.e. 56 per cent of the sample. In six of them the rotational peaks are among the 20 largest amplitude peaks. Moreover, we detect rotation peaks in 26 out of 34 main-sequence stars (76 per cent) or 16 out of 24 mainsequence stars falling within the general location of the Kepler $\delta$ Sct instability region (Fig. 1). In some cases, it seems that more than one spot could be present on the stellar surface (e.g. ID11). In other cases, like ID14, rotation seems to be almost synchronized with the orbital period in a binary system, similar to the cool rotating variable ID06. One star, ID28, seems to be a ROTD-type variable similar to those detected by Balona (2013): most likely, the sharp peak is the rotational frequency, and the hump is due to Rossby modes (Saio et al. 2018).

There is a clear indication of activity and star-spots on the surface of the A-type stars, as previously found by Balona (2011, 2013). This suggests the presence of magnetic fields on the stellar surfaces that are not expected in such hot stars with very thin convective envelopes. The dynamo mechanism, which is thought to be responsible for magnetic activity in cool stars, cannot operate in stars with predominantly radiative envelopes. Star-spots seem to be also common among the much hotter B stars (Balona 2016; Balona et al. 2019). This is not surprising since there is no difference in the envelope structure between the A and B stars.

Amplitude variations are found among the frequencies of highest amplitude in all four stars belonging to Group III (see last column of Table 1). They show a diversity of behaviours, including peaks with secular or sinusoidal variations. Similar results are obtained for all seven stars in Group II. The presence of amplitude variations supports the conclusions by Bowman et al. (2016), who analysed a sample of almost $1000 \delta$ Sct stars using Kepler LC data. They show that at least 60 per cent of these stars show amplitude modulation in at least one of the main pulsation frequencies. Nine stars in our sample (ID03, ID05, ID11, ID17, ID20, ID021, ID27, ID28, and ID33) were also analysed by Bowman et al. (2016).

Fig. 17 shows how the amplitude varies with time for two extreme cases in our sample (ID11 and ID19). ID11 is the only star where all 10 principal pulsation modes show significant amplitude

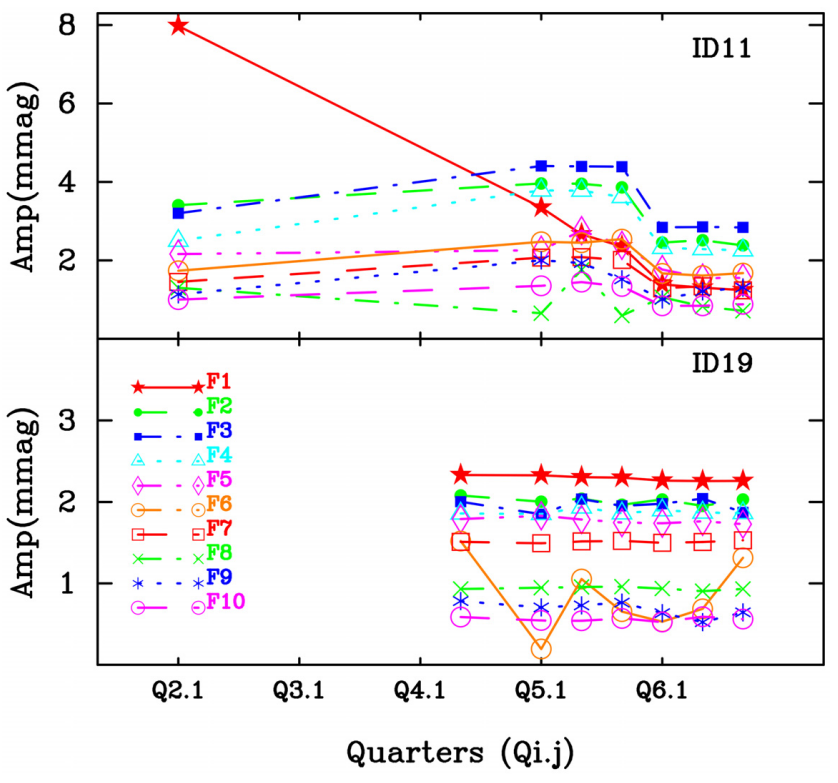

Figure 17. ID11 (top) and ID19 (bottom): temporal evolution of the amplitudes for the 10 main periodicities throughout the quarters with available SC mode photometry. ID11 shows variable amplitudes in all the 10 main pulsation modes. None of the main peaks in ID19 show significant amplitude variations, except F6 $=0.5246 \mathrm{~d}^{-1}$, which is identified as the stellar rotation frequency.

modulation, which agrees with the finding by Bowman et al. (2016). Interestingly, all the highest amplitude modes in ID11 seem to show a similar trend of amplitude with time, suggesting a common origin for the variability. On the contrary, ID19 is the only case where none of the 10 principal pulsation modes show significant amplitude variability (Fig. 17). However, small amplitude variations may be possible in some low-amplitude modes of this star, such as F13 and F17.

Nevertheless, it is important to note, when detecting amplitude modulation in a pulsation frequency, that its interpretation in terms of true amplitude variations cannot be definitely proven, as the interpretation in terms of unresolved closely spaced independent modes is always possible. A detailed study on the changes in amplitude and phase of the pulsation frequencies is needed to distinguish among the different possible scenarios.

Amplitude variability in $\delta$ Sct stars has important implications, not only for the interpretation of the pulsation mechanism, but also for the analysis and interpretation of the frequency content of the periodograms. A pulsation mode with variable amplitude is not well described by a simple sinusoid. Extraction of the corresponding peaks in the periodogram will lead to frequency residuals with lower amplitude, leading to a cascade effect of spurious frequencies as the pre-whitening amplitude decreases. This produces the same effect that the extraction of unresolved peaks due to the finite duration of a time series, as discussed by Balona (2014b). In effect, the only meaningful frequencies that may be extracted are those that are resolved or partially resolved in the periodogram of the raw data.

Analysis of the ID36 light curve shows that it is a binary system with a tidally distorted pulsating component and where reflection effects are important. The high-frequency region in the echelle diagram shows many equally spaced multiplets, which is likely the result of the variable viewing angle along the orbit. In principle, it should be possible to deduce the spherical harmonic, $l$, by counting the number of multiplets associated with a given pulsation mode (Balona 2018b), just as in the roAp stars. However, in roAp 
stars only axisymmetric modes are excited (for reasons that are not entirely clear), whereas in ID36, both axisymmetric and nonaxisymmetric modes may be seen. The visibility of tidally induced multiplets depends on the orbital inclination. Also complicating mode identification is the fact that the pulsations are unlikely to be described by a single spherical harmonic and the most relevant theory of Reyniers \& Smeyers (2003) assumes a circular orbit where the stars are in synchronous rotation (not seen here).

Flares have also been detected in the residual light curves of the binary system ID36, which probably arise from the pulsating $\delta$ Sct component. This would make ID36 one of the few pulsating stars in which flares have been detected. It is very difficult to identify flares in the rapid light variations due to pulsation. In fact, the flares of ID36 are not directly detectable in the original light curves, but only in the residuals light curves after removing the pulsations. Balona $(2012,2013,2015)$ finds that flares are visible in about 2.5 per cent of A stars, from the Kepler sample, though whether the flare originates on the A star itself or on a cool companion is a matter for debate (Pedersen et al. 2017). The main reason for attributing the flares to the A stars is that the flare energies are much larger than those observed in cool stars. Another indication is that a large fraction of A stars seem to have star-spots that would mean that flaring would not be unexpected. The flare energies in ID36 are about $10^{35}$ erg (Table 10) that are higher than the most energetic flares seen in cool dwarfs. Therefore, it is reasonable to attribute the flares origin in the A star and not the companion.

ID36, together with ID06 and ID07, is among the targets showing the most intense stellar activity in our sample, and the only in which we detect flares with the available data. Indeed, ID06 is the most active star in our sample and the one showing the strongest flares. This agrees well with the results by Balona (2015) regarding that it is more likely to detect flares in stars that are more active.

ID36 highlights the possibility of finding similar cases, i.e. $\delta$ Scttype pulsating stars with flares masked by oscillations. However, for now, ID36 is the only example and it seems to be a unique case, taking into account the current level of accuracy and availability of photometric time series. The nature of ID36, being in a binary system and belonging to an open cluster, together with the availability of 4 yr of Kepler LC and 18(+1) months of SC continuous monitoring, makes it a very interesting target to carry out a much deeper study in an upcoming work.

\section{ACKNOWLEDGEMENTS}

The authors thank an anonymous referee for careful reading and comments on our manuscript that helped us to improve it. This research was supported by the Dirección General de Investigación (DGI) under projects AYA2016-79425-C03-03-P, ESP2017-87676C05-02-R, and ESP2017-87143-R and the Centre of Excellence 'Severo Ochoa' Instituto de Astrofísica de Andalucía (SEV-20170709). LAB wishes to thank the South African Astronomical Observatory and the National Research Foundation for financial support. The data included in this paper were collected by the Kepler mission. Funding for the Kepler mission is provided by the NASA Science Mission directorate. The authors thank the Kepler team for their generosity in releasing these data and for their outstanding efforts that have made these results possible. All the data presented in this work were obtained from the Mikulski Archive for Space Telescopes (MAST) at the Space Telescope Science Institute (STScI). STScI is operated by the Association of Universities for Research in Astronomy, Inc., under NASA contract NASA-26555. Support for MAST for non-HST data is provided by the NASA
Office of Space Science via grant NNX09AF08G and by other grants and contracts. This work made also use of the SIMBAD data base, operated at CDS, Strasbourg, France.

\section{REFERENCES}

Balona L. A., 2011, MNRAS, 415, 1691

Balona L. A., 2012, MNRAS, 423, 3420

Balona L. A., 2013, MNRAS, 431, 2240 (Ba13)

Balona L. A., 2014a, MNRAS, 437, 1476

Balona L. A., 2014b, MNRAS, 439, 3453

Balona L. A., 2014c, MNRAS, 441, 3543

Balona L. A., 2014d, MNRAS, 443, 1946 (Ba14)

Balona L. A., 2015, MNRAS, 447, 2714 (Ba15)

Balona L. A., 2016, MNRAS, 457, 3724

Balona L. A., 2017, MNRAS, 467, 1830

Balona L. A., 2018a, MNRAS, 475, 359

Balona L. A., 2018b, MNRAS, 476, 4840

Balona L. A., 2018c, MNRAS, 479, 183

Balona L. A., Daszyńska-Daszkiewicz J., Pamyatnykh A. A., 2015, MNRAS, 452, 3073

Balona L. A. et al., 2016, MNRAS, 460, 1318

Balona L. A. et al., 2019, MNRAS, 485, 3457

Benz A. O., Güdel M., 2010, ARA\&A, 48, 241

Borucki W. J. et al., 2010, Science, 327, 977

Bowman D. M., Kurtz D. W., Breger M., Murphy S. J., Holdsworth D. L., 2016, MNRAS, 460, 1970

Chapellier E. et al., 2011, A\&A, 525, A23

Claret A., 2004, A\&A, 424, 919

Corsaro E. et al., 2012, ApJ, 757, 190

Curtis J. L., Agüeros M. A., Douglas S. T., Meibom S., 2019, ApJ, 879, 49

Debosscher J., Blomme J., Aerts C., De Ridder J., 2011, A\&A, 529, A89 (De11)

Dias W. S., Monteiro H., Caetano T. C., Lépine J. R. D., Assafin M., Oliveira A. F., 2014, A\&A, 564, A79

Dupret M., Grigahcène A., Garrido R., Gabriel M., Scuflaire R., 2004, A\&A, 414, L17

Gaia Collaboration et al., 2016, A\&A, 595, A1

Gaia Collaboration et al., 2018, A\&A, 616, A1

García Hernández A. et al., 2009, A\&A, 506, 79

García Hernández A. et al., 2013, A\&A, 559, A63

Grigahcène A., Dupret M., Gabriel M., Garrido R., Scuflaire R., 2005, A\&A, 434,1055

Guzik J. A., Kaye A. B., Bradley P. A., Cox A. N., Neuforge C., 2000, ApJ, 542, L57

Janes K., Barnes S. A., Meibom S., Hoq S., 2013, AJ, 145, 7

Kallinger T., Reegen P., Weiss W. W., 2008, A\&A, 481, 571

Kharchenko N. V., Piskunov A. E., Schilbach E., Röser S., Scholz R. D., 2013, A\&A, 558, A53

Koch D. G. et al., 2010, ApJ, 713, L79

Lenz P., Breger M., 2005, Commun. Asteroseismol., 146, 53

Luo Y. P., Zhang X. B., Luo C. Q., Deng L. C., Luo Z. Q., 2009, New Astron., 14, 584 (Lu09)

McQuillan A., Mazeh T., Aigrain S., 2014, ApJS, 211, 24 (Mc14)

Marigo P. et al., 2017, ApJ, 835, 77

Mathur S. et al., 2017, ApJS, 229, 30 (Ma17)

Meibom S. et al., 2011, ApJ, 733, L9 (Me11)

Molenda-Żakowicz J., Brogaard K., Niemczura E., Bergemann M., Frasca A., Arentoft T., Grundahl F., 2014, MNRAS, 445, 2446 (Mo14)

Nielsen M. B., Gizon L., Schunker H., Karoff C., 2013, A\&A, 557, L10 (Ni13)

Pamyatnykh A. A., 2000, in Breger M., Montgomery M., eds, ASP Conf. Ser. Vol. 210, Delta Scuti and Related Stars. Astron. Soc. Pac., San Francisco, p. 215

Pecaut M. J., Mamajek E. E., 2013, ApJS, 208, 9

Pedersen M. G., Antoci V., Korhonen H., White T. R., Jessen-Hansen J., Lehtinen J., Nikbakhsh S., Viuho J., 2017, MNRAS, 466, 3060 
Peña J. H., Fox Machado L., García H., Rentería A., Skinner S., Espinosa A., Romero E., 2011, Rev. Mex. Astron. Astrofis., 47, 309

Reegen P., 2007, A\&A, 467, 1353

Reinhold T., Gizon L., 2015, A\&A, 583, A65 (Re15)

Reinhold T., Reiners A., Basri G., 2013, A\&A, 560, A4 (Re13)

Reyniers K., Smeyers P., 2003, A\&A, 404, 1051

Rodríguez E., Rodríguez-López C., López-González M. J., Amado P. J., Ocando S., Berdiñas Z. M., 2016, MNRAS, 457, 1851

Saio H., Kurtz D. W., Murphy S. J., Antoci V. L., Lee U., 2018, MNRAS, 474,2774

Sanders W. L., 1971, A\&A, 15, 368

Sandquist E. L. et al., 2016, ApJ, 831, 11 (Sa16)

Shibahashi H., Kurtz D. W., 2012, MNRAS, 422, 738

Stello D. et al., 2011a, ApJ, 737, L10

Stello D. et al., 2011b, ApJ, 739, 13 (St11)

Uytterhoeven K. et al., 2011, A\&A, 534, A125 (Uy11)

van Cauteren P., Lampens P., Robertson C. W., Strigachev A., 2005, Commun. Asteroseismol., 146, 21

Walkowicz L. M. et al., 2011, AJ, 141, 50

Xiong D. R., Deng L., Zhang C., Wang K., 2016, MNRAS, 457, 3163

Yontan T. et al., 2015, Ap\&SS, 355, 267

Zacharias N., Finch C. T., Girard T. M., Henden A., Bartlett J. L., Monet D. G., Zacharias M. I., 2013, AJ, 145, 44

\section{APPENDIX A: GAIA LUMINOSITIES AND MEMBERSHIP}

Table A1 lists the proper motions and parallaxes from Gaia DR2 (Gaia Collaboration et al. 2016, 2018) for our sample of stars. These parameters are available for all except four targets. These parallaxes are used to determine the luminosities of the targets. From the Gaia DR2 parallax $\varpi$, the absolute magnitude can be calculated using $M_{V}=V_{0}+5\left(\log _{10} \varpi+1\right)$, where $V_{0}$ is the reddening-free $V$ magnitude. We used $V$ magnitudes from SIMBAD and a reddening correction of $A_{V}=0.15 \pm 0.02 \mathrm{mag}$ (Curtis et al. 2019). The absolute bolometric magnitude is given by $M_{\mathrm{bol}}=M_{V}+\mathrm{BC}_{V}$, where $\mathrm{BC}_{V}$ is the bolometric correction in $V$ and $M_{\text {bol } \odot}=4.74 \mathrm{mag}$ is the absolute bolometric magnitude of the Sun. The bolometric correction as a function of $T_{\text {eff }}$ is given in Pecaut \& Mamajek (2013). Finally, the luminosity relative to the Sun is found using $\log L / \mathrm{L}_{\odot}=$ $-0.4\left(M_{\mathrm{bol}}-M_{\mathrm{bol} \odot}\right)$. From the error in the Gaia DR2 parallax, the typical standard deviation in $\log \left(L / \mathrm{L}_{\odot}\right)$ is estimated to be about 0.05 dex, allowing for a standard deviations of $0.01 \mathrm{mag}$ in the apparent magnitude, $0.10 \mathrm{mag}$ in visual extinction, and $0.02 \mathrm{mag}$ in the bolometric correction in addition to the parallax error.

Table A1. Data for the sample of stars. ID, KIC: identification numbers in this work and from the Kepler Input Catalogue (KIC); $\mu_{\alpha} \cos \delta, \mu_{\delta}, \varpi:$ proper motions and parallax from Gaia DR2; V: apparent magnitude from SIMBAD. Luminosities are from Gaia parallaxes assuming $A_{V}=0.15$ mag. The last column is the membership using the criterion described in the text $(\mathrm{M}=$ member, $\mathrm{N}=$ non-member $)$.

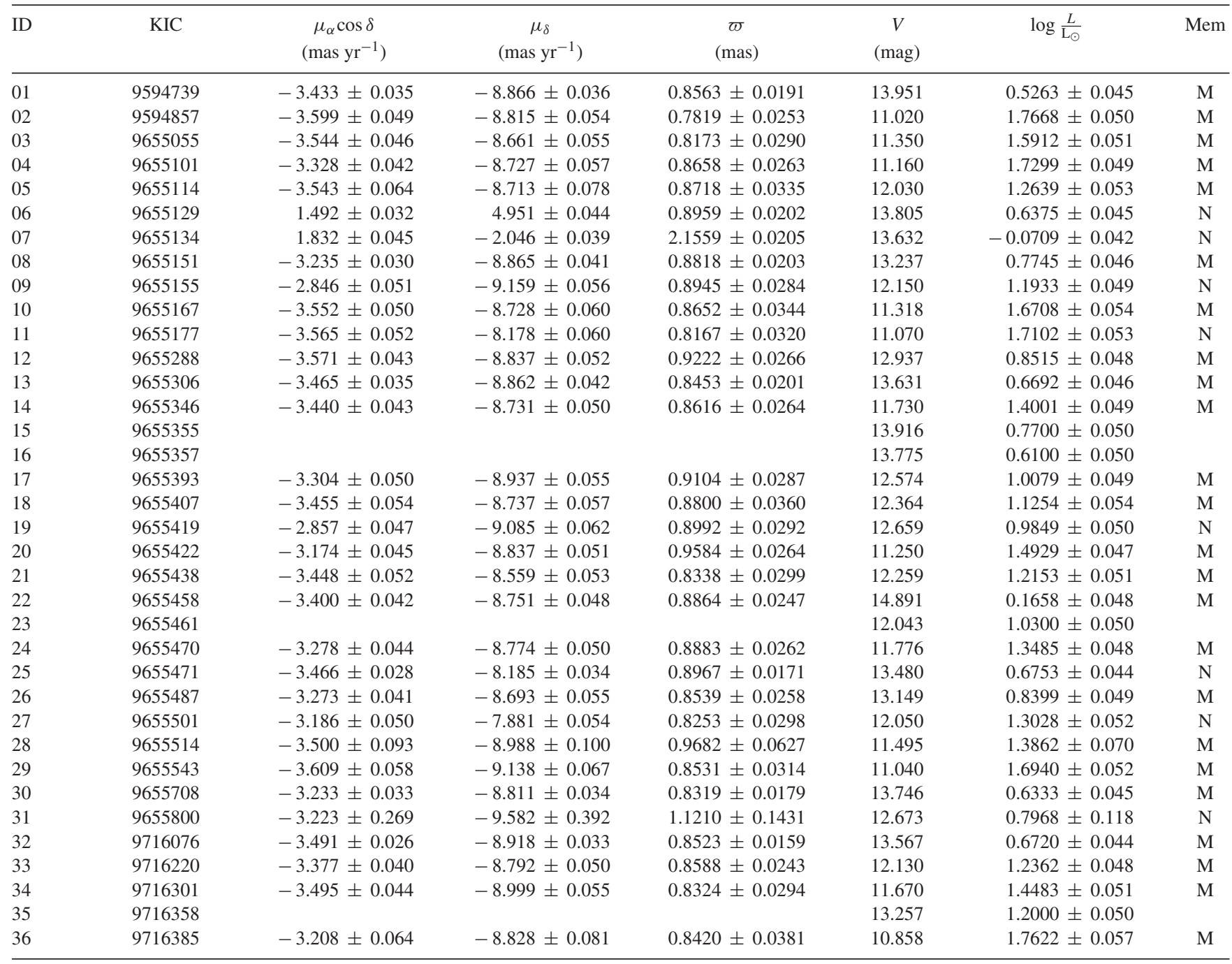


Moreover, we follow the method of Curtis et al. (2019) in using the Gaia DR2 parallax to identify members. The median proper

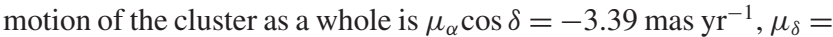

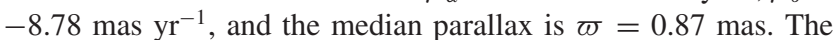
criterion for membership is that the proper motion must be within 0.5 mas $\mathrm{yr}^{-1}$ of the mean cluster proper motion and the parallax within 0.3 mas of the cluster median. The results are listed in the last column of Table A1.

\section{APPENDIX B: RESULTS OF THE FOURIER ANALYSIS FOR THE MAIN-SEQUENCE PULSATORS}

Tables B1-B3 list the results of the Fourier fits for the 20 main significant peaks, when possible, for each of the main-sequence pulsators in our sample.

Table B1. Fourier fit for the 20 main significant peaks, when possible, for the $\delta$ Sct- and $\gamma$ Dor-type stars of Group I. The quarters Qi.j are indicated; S/N are the amplitude signal-to-noise ratios; the residuals $\sigma_{n}$, in mmag, after removing the $n$ first peaks are also indicated.

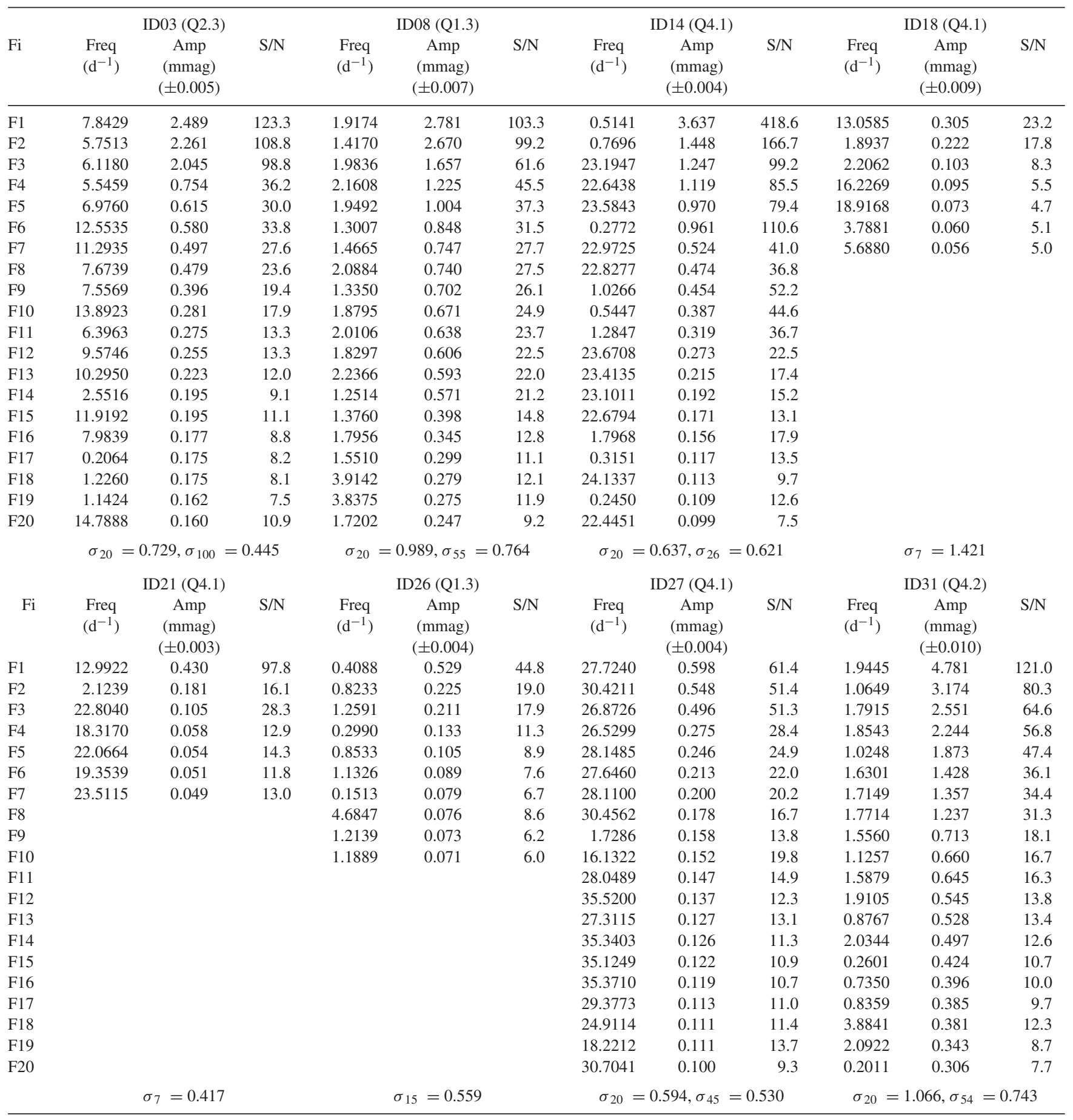


Table B1 - continued

\begin{tabular}{|c|c|c|c|c|c|c|}
\hline \multirow[b]{2}{*}{$\mathrm{Fi}$} & \multicolumn{3}{|c|}{ ID34 (Q2.3) } & \multicolumn{3}{|c|}{ ID35 (Q2.1) } \\
\hline & $\begin{array}{c}\text { Freq } \\
\left(d^{-1}\right)\end{array}$ & $\begin{array}{c}\text { Amp } \\
(\mathrm{mmag}) \\
( \pm 0.002)\end{array}$ & $\mathrm{S} / \mathrm{N}$ & $\begin{array}{l}\text { Freq } \\
\left(d^{-1}\right)\end{array}$ & $\begin{array}{c}\text { Amp } \\
(\mathrm{mmag}) \\
( \pm 0.006)\end{array}$ & $\mathrm{S} / \mathrm{N}$ \\
\hline $\mathrm{F} 1$ & 0.6477 & 0.075 & 11.3 & 0.6186 & 0.593 & 29.0 \\
\hline F2 & 0.8820 & 0.048 & 7.3 & 0.2730 & 0.426 & 20.8 \\
\hline F3 & 0.3796 & 0.033 & 5.0 & 0.8058 & 0.356 & 17.4 \\
\hline $\mathrm{F} 4$ & 0.9318 & 0.033 & 5.0 & 0.6452 & 0.348 & 17.0 \\
\hline F5 & & & & 0.4873 & 0.306 & 15.0 \\
\hline F6 & & & & 0.6954 & 0.291 & 14.2 \\
\hline F7 & & & & 0.1380 & 0.253 & 12.4 \\
\hline F8 & & & & 0.5541 & 0.252 & 12.3 \\
\hline F9 & & & & 0.9088 & 0.243 & 11.9 \\
\hline F10 & & & & 1.5184 & 0.199 & 9.7 \\
\hline F11 & & & & 0.7714 & 0.191 & 9.3 \\
\hline F12 & & & & 1.4217 & 0.188 & 9.2 \\
\hline F13 & & & & 0.3745 & 0.183 & 8.9 \\
\hline F14 & & & & 1.9110 & 0.152 & 7.4 \\
\hline F15 & & & & 0.5813 & 0.151 & 7.4 \\
\hline \multicolumn{7}{|l|}{ F16 } \\
\hline \multicolumn{7}{|l|}{ F17 } \\
\hline \multicolumn{7}{|l|}{ F18 } \\
\hline \multicolumn{7}{|l|}{ F19 } \\
\hline \multicolumn{7}{|l|}{ F20 } \\
\hline & & $4=0.334$ & & & $15=0.809$ & \\
\hline
\end{tabular}

Table B2. Fourier fit for the 20 main significant peaks for the $\delta$ Sct stars of Group II. The quarters Qi.j corresponding to each fit are indicated.

\begin{tabular}{|c|c|c|c|c|c|c|c|c|c|c|c|c|}
\hline \multirow[b]{2}{*}{$\mathrm{Fi}$} & \multicolumn{3}{|c|}{ ID11 (Q2.1) } & \multicolumn{3}{|c|}{ ID12 (Q4.2) } & \multicolumn{3}{|c|}{ ID17 (Q4.1) } & \multicolumn{3}{|c|}{ ID19 (Q4.2) } \\
\hline & $\begin{array}{c}\text { Freq } \\
\left(d^{-1}\right)\end{array}$ & $\begin{array}{c}\text { Amp } \\
(\mathrm{mmag}) \\
( \pm 0.011)\end{array}$ & $\mathrm{S} / \mathrm{N}$ & $\begin{array}{c}\text { Freq } \\
\left(d^{-1}\right)\end{array}$ & $\begin{array}{c}\text { Amp } \\
(\mathrm{mmag}) \\
( \pm 0.011)\end{array}$ & $\mathrm{S} / \mathrm{N}$ & $\begin{array}{c}\text { Freq } \\
\left(\mathrm{d}^{-1}\right)\end{array}$ & $\begin{array}{c}\text { Amp } \\
(\mathrm{mmag}) \\
( \pm 0.009)\end{array}$ & $\mathrm{S} / \mathrm{N}$ & $\begin{array}{l}\text { Freq } \\
\left(d^{-1}\right)\end{array}$ & $\begin{array}{c}\text { Amp } \\
(\mathrm{mmag}) \\
( \pm 0.012)\end{array}$ & $\mathrm{S} / \mathrm{N}$ \\
\hline F1 & 8.5096 & 7.975 & 205.3 & 14.4973 & 2.136 & 101.6 & 28.1592 & 3.935 & 192.6 & 14.6011 & 2.330 & 85.0 \\
\hline $\mathrm{F} 2$ & 7.4581 & 3.407 & 86.8 & 16.6125 & 1.410 & 59.8 & 30.9478 & 3.876 & 168.1 & 12.9208 & 2.079 & 76.8 \\
\hline F3 & 7.8223 & 3.201 & 81.1 & 20.7368 & 1.350 & 55.3 & 14.6361 & 2.278 & 137.9 & 26.7453 & 2.003 & 65.9 \\
\hline $\mathrm{F} 4$ & 3.4230 & 2.502 & 74.3 & 17.3785 & 1.067 & 43.5 & 13.3017 & 2.222 & 137.5 & 14.8745 & 1.858 & 67.7 \\
\hline F6 & 7.4102 & 1.735 & 44.3 & 30.1574 & 0.834 & 28.9 & 26.2090 & 2.111 & 113.6 & 0.5246 & 1.519 & 41.3 \\
\hline F7 & 8.3734 & 1.452 & 37.3 & 23.1657 & 0.777 & 31.4 & 23.4051 & 2.066 & 129.8 & 11.8006 & 1.510 & 55.3 \\
\hline F8 & 9.2536 & 1.302 & 34.1 & 25.3535 & 0.774 & 29.6 & 16.6544 & 1.999 & 117.2 & 8.7809 & 0.931 & 32.6 \\
\hline F9 & 7.0995 & 1.135 & 29.3 & 28.4447 & 0.604 & 21.6 & 33.6824 & 1.873 & 79.5 & 21.5611 & 0.782 & 26.0 \\
\hline F10 & 8.5813 & 1.001 & 25.8 & 27.2901 & 0.547 & 20.0 & 23.0574 & 1.698 & 108.9 & 23.7605 & 0.587 & 19.3 \\
\hline F11 & 6.8732 & 0.854 & 22.2 & 15.5350 & 0.526 & 23.6 & 14.9610 & 1.119 & 67.4 & 19.4524 & 0.567 & 19.6 \\
\hline F12 & 5.8293 & 0.821 & 22.2 & 23.7116 & 0.491 & 19.6 & 29.0191 & 0.810 & 38.1 & 26.3104 & 0.566 & 18.6 \\
\hline F17 & 2.9112 & 0.466 & 14.1 & 27.6081 & 0.430 & 15.6 & 13.7133 & 0.484 & 29.8 & 16.2365 & 0.467 & 16.9 \\
\hline F18 & 6.5614 & 0.463 & 12.2 & 24.2873 & 0.422 & 16.6 & 24.2151 & 0.482 & 28.9 & 0.9240 & 0.454 & 12.3 \\
\hline F19 & 11.1020 & 0.437 & 11.9 & 29.2753 & 0.422 & 14.8 & 15.3213 & 0.459 & 27.5 & 10.4649 & 0.414 & 14.9 \\
\hline \multirow[t]{2}{*}{$\mathrm{F} 20$} & 6.3452 & 0.435 & 11.5 & 30.7119 & 0.380 & 13.0 & 34.2425 & 0.401 & 17.4 & 21.9131 & 0.373 & 12.4 \\
\hline & \multicolumn{3}{|c|}{$\sigma_{20}=1.543, \sigma_{100}=0.924$} & \multicolumn{3}{|c|}{$\sigma_{20}=1.535, \sigma_{100}=0.939$} & \multicolumn{3}{|c|}{$\sigma_{20}=1.293, \sigma_{100}=0.820$} & \multicolumn{3}{|c|}{$\sigma_{20}=1.496, \sigma_{100}=0.924$} \\
\hline
\end{tabular}


Table B2 - continued

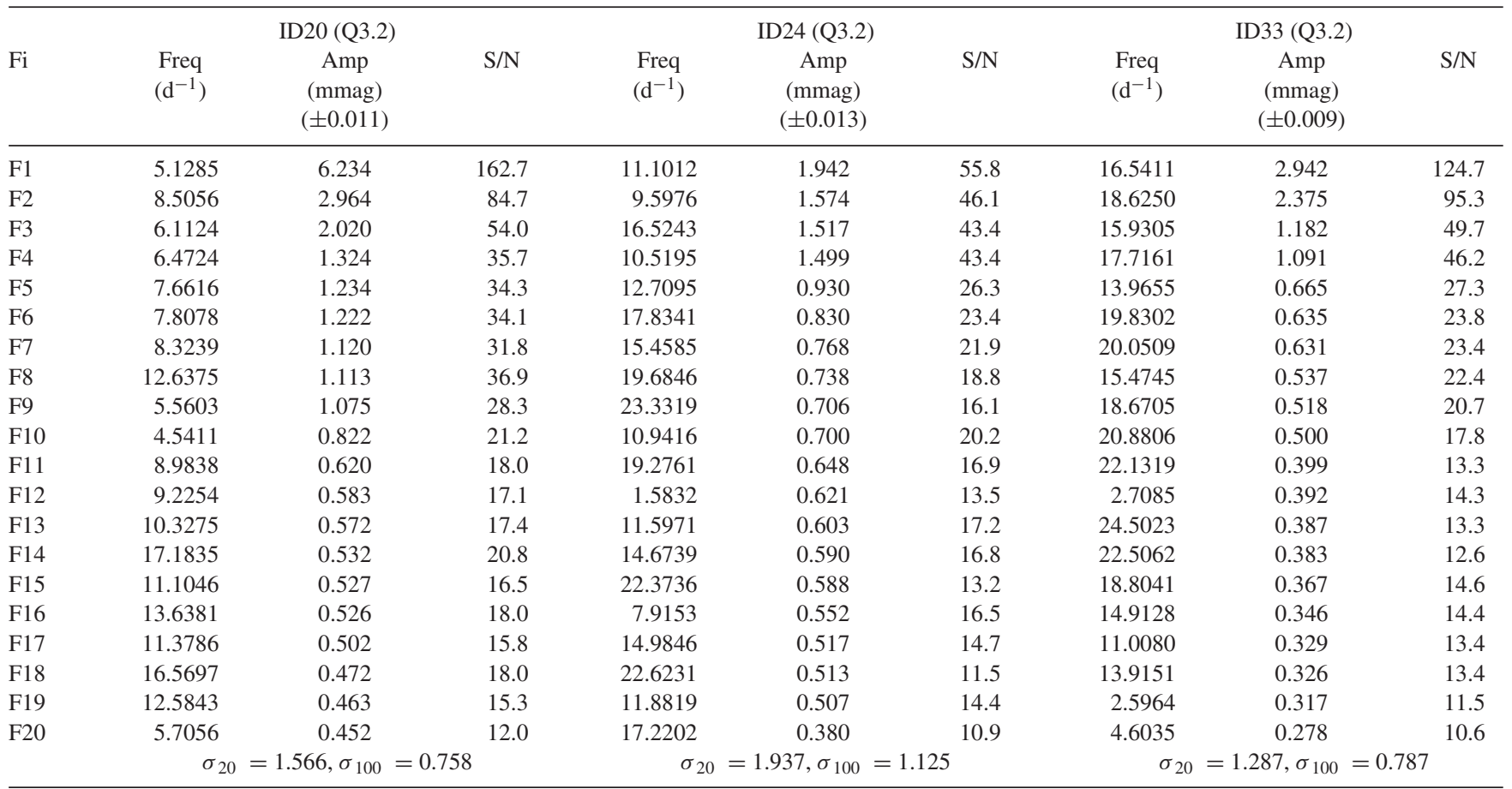

Table B3. Fourier fit for the 20 main significant peaks for the $\delta$ Sct stars of Group III. The quarters Qi.j corresponding to each fit are indicated. Some identifications are given for ID36.

\begin{tabular}{|c|c|c|c|c|c|c|c|c|c|c|c|c|c|}
\hline \multirow[b]{2}{*}{$\mathrm{Fi}$} & \multicolumn{3}{|c|}{ ID02 (Q2.1) } & \multicolumn{3}{|c|}{ ID05 (Q2.3) } & \multicolumn{3}{|c|}{ ID28 (Q1.3) } & \multicolumn{4}{|c|}{ ID36 (Q2.3) } \\
\hline & $\begin{array}{c}\text { Freq } \\
\left(d^{-1}\right)\end{array}$ & $\begin{array}{c}\text { Amp } \\
(\mathrm{mmag}) \\
( \pm 0.021)\end{array}$ & $\mathrm{S} / \mathrm{N}$ & $\begin{array}{c}\text { Freq } \\
\left(d^{-1}\right)\end{array}$ & $\begin{array}{c}\text { Amp } \\
(\mathrm{mmag}) \\
( \pm 0.009)\end{array}$ & $\mathrm{S} / \mathrm{N}$ & $\begin{array}{c}\text { Freq } \\
\left(d^{-1}\right)\end{array}$ & $\begin{array}{c}\text { Amp } \\
(\mathrm{mmag}) \\
( \pm 0.005)\end{array}$ & $\mathrm{S} / \mathrm{N}$ & $\begin{array}{c}\text { Freq } \\
\left(d^{-1}\right)\end{array}$ & $\begin{array}{c}\text { Amp } \\
(\mathrm{mmag}) \\
( \pm 0.004)\end{array}$ & $\mathrm{S} / \mathrm{N}$ & Iden \\
\hline F1 & 9.9558 & 29.253 & 364.5 & 20.5683 & 4.839 & 209.3 & 15.1762 & 4.677 & 297.6 & 21.6131 & 5.066 & 606.6 & \\
\hline $\mathrm{F} 2$ & 6.0546 & 9.293 & 115.3 & 23.9178 & 3.365 & 129.9 & 12.5403 & 2.092 & 155.3 & 0.1900 & 2.690 & 423.0 & $v_{\text {orb }}$ \\
\hline F3 & 7.4453 & 6.548 & 79.7 & 10.7244 & 1.538 & 70.9 & 14.6005 & 1.623 & 106.6 & 19.0216 & 2.237 & 298.9 & \\
\hline F6 & 4.2174 & 3.431 & 43.7 & 22.8478 & 0.787 & 32.5 & 13.9725 & 0.735 & 50.0 & 0.8616 & 0.579 & 91.0 & $v_{\text {rot }}$ \\
\hline F7 & 7.4003 & 3.097 & 37.7 & 22.5580 & 0.733 & 30.8 & 13.7356 & 0.685 & 47.3 & 20.1400 & 0.673 & 85.7 & \\
\hline F8 & 5.6875 & 2.468 & 30.8 & 16.1507 & 0.676 & 31.2 & 15.3526 & 0.459 & 28.9 & 0.3789 & 0.542 & 83.8 & $2 v_{\text {orb }}$ \\
\hline F9 & 8.4922 & 1.932 & 23.7 & 18.5183 & 0.673 & 29.9 & 15.8322 & 0.424 & 26.1 & 20.3265 & 0.400 & 50.6 & $\mathrm{~F} 7+v_{\text {orb }}$ \\
\hline F10 & 5.0907 & 1.909 & 24.0 & 9.2361 & 0.503 & 22.1 & 18.3904 & 0.395 & 22.9 & 19.9529 & 0.394 & 50.5 & F7- $v_{\text {orb }}$ \\
\hline F11 & 6.6838 & 1.861 & 22.9 & 23.8794 & 0.449 & 17.4 & 14.7758 & 0.353 & 22.9 & 0.8329 & 0.513 & 80.8 & $v_{\text {rot2 }}$ \\
\hline F17 & 6.2283 & 1.208 & 14.9 & 13.5609 & 0.296 & 14.2 & 17.1792 & 0.285 & 16.4 & 21.8056 & 0.252 & 30.0 & $\mathrm{~F} 1+v_{\text {orb }}$ \\
\hline F18 & 19.9119 & 1.124 & 33.0 & 26.1526 & 0.284 & 9.7 & 15.7732 & 0.246 & 15.2 & 21.7266 & 0.251 & 29.9 & \\
\hline F19 & 5.3240 & 1.104 & 13.8 & 22.4086 & 0.277 & 11.7 & 14.1437 & 0.234 & 15.8 & 19.2104 & 0.226 & 29.9 & $\mathrm{~F} 3+v_{\text {orb }}$ \\
\hline \multirow[t]{2}{*}{ F20 } & 4.6547 & 1.030 & 13.0 & 24.7243 & 0.272 & 10.0 & 18.0422 & 0.218 & 12.5 & 21.8958 & 0.203 & 24.1 & \\
\hline & \multicolumn{3}{|c|}{$\sigma_{20}=2.961, \sigma_{100}=1.493$} & \multicolumn{3}{|c|}{$\sigma_{20}=1.201, \sigma_{100}=0.792$} & \multicolumn{3}{|c|}{$\sigma_{20}=0.832, \sigma_{100}=0.499$} & \multicolumn{3}{|c|}{$\sigma_{20}=0.545, \sigma_{100}=0.281$} & \\
\hline
\end{tabular}

This paper has been typeset from a $\mathrm{TE}_{\mathrm{E}} / \mathrm{L} \mathrm{T} \mathrm{T} \mathrm{X}$ file prepared by the author. 